\title{
Fast Kalman filtering on quasilinear dendritic trees
}

\author{
Liam Paninski \\ Department of Statistics and Center for Theoretical Neuroscience \\ Columbia University \\ http://www.stat.columbia.edu/ liam
}

November 3, 2009

\begin{abstract}
Optimal filtering of noisy voltage signals on dendritic trees is a key problem in computational cellular neuroscience. However, the state variable in this problem - the vector of voltages at every compartment - is very high-dimensional: realistic multicompartmental models often have on the order of $N=10^{4}$ compartments. Standard implementations of the Kalman filter require $O\left(N^{3}\right)$ time and $O\left(N^{2}\right)$ space, and are therefore impractical. Here we take advantage of three special features of the dendritic filtering problem to construct an efficient filter: (1) dendritic dynamics are governed by a cable equation on a tree, which may be solved using sparse matrix methods in $O(N)$ time; and current methods for observing dendritic voltage (2) provide low SNR observations and (3) only image a relatively small number of compartments at a time. The idea is to approximate the Kalman equations in terms of a low-rank perturbation of the steady-state (zero-SNR) solution, which may be obtained in $O(N)$ time using methods that exploit the sparse tree structure of dendritic dynamics. The resulting methods give a very good approximation to the exact Kalman solution, but only require $O(N)$ time and space. We illustrate the method with applications to real and simulated dendritic branching structures, and describe how to extend the techniques to incorporate spatially subsampled, temporally filtered, and nonlinearly transformed observations.
\end{abstract}

\section{Introduction}

The problem of understanding dendritic computation remains a key open challenge in cellular and computational neuroscience (Stuart et al., 1999; Spruston, 2008; Sjostrom et al., 2008). The major difficulty is in recording physiological signals (especially voltage) with sufficient spatiotemporal resolution on dendritic trees. As pointed out in (Morse et al., 2001; Wood et al., 2004; Huys et al., 2006), if we are given the full spatiotemporal voltage signal on the dendritic tree, then simple, direct statistical methods allow us to infer many biophysical quantities of interest, including passive cable parameters (e.g., the axial resistance at each point on the tree), active properties (e.g., the spatial membrane density distribution of voltage-gated channels), and in some cases even time-varying information (e.g., synaptic weights and presynaptic input conductances (Cox, 2004; Paninski and Ferreira, 2008; Paninski et al., 2009)). Unfortunately, multiple-electrode recordings from dendrites are quite technically challenging, and provide spatially-incomplete observations (Stuart and Sakmann, 1994; Cox and Griffith, 2001; Cox and Raol, 2004; Bell and Craciun, 2005; Petrusca et al., 2007), while high-resolution imaging techniques provide more spatially-complete observations, but with significantly lower 
signal-to-noise (Djurisic et al., 2004; Sacconi et al., 2006; Araya et al., 2006; Palmer and Stuart, 2006; Gobel and Helmchen, 2007; Vucinic and Sejnowski, 2007; Canepari et al., 2007; Djurisic et al., 2008).

One avenue for extending the reach of these currently available methods is to develop statistical techniques for optimally combining, filtering, and deconvolving these noisy signals. As argued in (Huys and Paninski, 2009), state-space filtering methods are attractive here, since these methods allow us to quite transparently incorporate 1) realistic, spatially-complex multicompartmental models of dendritic dynamics and 2) time-varying, heterogeneous observations (e.g., spatially-scanned multiphoton imaging data) into our filtering equations.

The problem is that the time-varying state vector in this problem - which includes, at least, the vector of voltages at every compartment — is very high-dimensional: realistic multicompartmental models often have on the order of $N \sim 10^{4}$ compartments. Standard implementations of state-space filter methods (e.g., the Kalman filter) require $O\left(N^{3}\right)$ time and $O\left(N^{2}\right)$ space, and are therefore impractical for applications to large dendritic trees. (Note that the analyses in (Huys and Paninski, 2009) were restricted to relatively low-dimensional systems.)

However, we may take advantage of three special features of the dendritic filtering problem to construct efficient filtering methods. First, dendritic dynamics are governed by a cable equation on a tree, which may be solved using symmetric sparse matrix methods in $O(N)$ time (Hines, 1984). Second, current methods for imaging dendritic voltage provide low SNR observations, as discussed above. Finally, in typical experiments we record only a few image observations ( $n<100$ or so coarse pixels) at a time. Taken together, these special features allow us to approximate the Kalman equations in terms of a low-rank perturbation of the steady-state (zero-SNR) solution, which in turn may be obtained in $O(N)$ time using efficient matrix solving methods that exploit the sparse tree structure of the dynamics ${ }^{1}$. The resulting methods provide a very good approximation to the exact Kalman solution, but only require $O(N)$ time and space. Some $O\left(n^{3}\right)$ operations are required; thus $n$ is the real bottleneck for this problem, not $N$.

We present a derivation of this fast tree Kalman filter method in section 2 below. We illustrate the method with some applications to simulated, spatially-subsampled observations on large, real dendritic trees in section 3. Finally, in section 4 we discuss a number of key extensions of the basic method: in particular, we can incorporate spatially blurred or scanned observations; temporally filtered observations and inhomogenous noise sources on the tree; "quasi-active" membrane dynamics (Koch, 1984; Manwani and Koch, 1999; Coombes et al., 2007; Kellems et al., 2009); and even in some cases nonlinear observations of the membrane state. Conclusions and directions for future research are discussed in section 5.

\section{Basic method}

We begin by describing the state-space model employed here. Most of our analysis is based on a simple linear-Gaussian (Kalman) model for the noisy dynamics of the dendritic voltage and for the observed data (though we will consider more general nonlinear observations in

\footnotetext{
${ }^{1}$ (Kellems et al., 2009) recently introduced powerful model-reduction methods for decreasing the effective dimensionality of the state vector in large dendritic voltage simulations; these methods are distinct from the techniques we introduce in this paper, but there are a number of interesting connections between these methods which we will describe below.
} 
section 4.3 below). This linear-Gaussian model is of course a crude approximation, but serves as a reasonable starting point for understanding subthreshold (non-spiking) passive dynamics along dendritic trees; see (Huys and Paninski, 2009) for further background and discussion. We take the state vector here to include the vector of voltages on the full dendritic tree: if we have broken up the tree into $N$ compartments, then our hidden state $V_{t}$ has dimensionality $N$. (See section 4.2 for a generalization.) For the dynamics and observation equations, we take

$$
\begin{aligned}
V_{t+d t} & =A V_{t}+i_{t} d t+\epsilon_{t}, \epsilon_{t} \sim \mathcal{N}\left(0, \sigma^{2} d t I\right) \\
y_{t} & =B_{t} V_{t}+\eta_{t}, \eta_{t} \sim \mathcal{N}\left(\mu_{t}^{y}, W_{t}\right)
\end{aligned}
$$

Here $A$ is an $N \times N$ matrix that implements the cable equation (as discussed further below), $i_{t}$ is an $N$-vector of known, possibly time-varying currents that are injected into the compartments, and $\epsilon_{t}$ denotes a Gaussian noise source that perturbs the cable equation stochastically on each time step; $\mathcal{N}(\mu, C)$ denotes a Gaussian density of mean $\mu$ and covariance $C$. We assume for now that the covariance of $\epsilon_{t}$ is proportional to the identity matrix, though we may generalize this assumption somewhat; see section 4.2 below. Note that it is fair to assume that membrane channel noise on a dendrite is spatially uncorrelated (corresponding to a diagonal covariance matrix for $\epsilon_{t}$ ); synaptic noise, on the other hand, may not be completely spatially uncorrelated between compartments (due to branching presynaptic axons forming multiple terminals on the observed postsynaptic cell), but modeling this source of noise as uncorrelated as well seems to be a reasonable first approximation. The noise is assumed to have zero mean (with no loss of generality, due to the inclusion of the $i_{t}$ term) scaled by a standard deviation $\sigma$ and a $\sqrt{d t}$ factor to ensure that the behavior of the model is insensitive to the choice of the timestep $d t$.

The second equation determines the observed data vector $y_{t}$. $B_{t}$ is a matrix that specifies how the observations are related instantaneously to the voltage vector ${ }^{2}$; for example, in the case of a whole-cell recording with a single electrode, $B_{t}$ would be an $N$ vector all of zeros, except at the compartment whose voltage is being recorded, while in an imaging experiment $B_{t}$ could encode instantaneous optical blurring and sampling transforming the $N$-element voltage vector $V_{t}$ to the $n$-pixel observed vector $y_{t}$. More generally, we could combine imaging and electrode observations, simply by appending the corresponding rows of $B_{t}$. It is also worth noting that our methods are sufficiently general to allow the observation gain matrix $B_{t}$, offset mean $\mu_{t}^{y}$, and noise covariance $W_{t}$ to vary with time; in fact, even the dimensionality of $y_{t}$ could vary with time (e.g., in the case of scanning optical experiments). However, neither $A$ nor $\sigma$ in the dynamics equation are allowed to vary with time.

We will begin by discussing a forward Euler discretization for the cable equation matrix $A$ :

$$
V_{t+d t}(x)=V_{t}(x)+d t\left(-g_{x} V_{t}(x)+i_{t}(x)+\sum_{w \in N(x)} a_{x w}\left[V_{t}(w)-V_{t}(x)\right]\right)+\epsilon_{t}(x),
$$

where $V_{t}(x)$ denotes the voltage in compartment $x$ at time $t, g_{x}$ is the membrane conductance in compartment $x, i_{t}(x)$ and $\epsilon_{t}(x)$ represent the known current and noise current, respectively, injected into compartment $x$ at time $t$, and $N(x)$ is the set of nearest neighbors of

\footnotetext{
${ }^{2}$ In some cases it is overly crude to assume that the observations $y_{t}$ are linearly and instantaneouly related to the voltage $V_{t}$; we will discuss extensions to this basic setup in section 4 below.
} 
compartment $x$, i.e., the set of all compartments adjoining $x^{3} ; a_{x w}=a_{w x}$ denotes the intracellular conductance between compartments $x$ and $w$. The choice of forward Euler here is for simplicity only, to keep the derivations below as transparent as possible; we will discuss more efficient implicit implementations of the discrete-time cable equation in section 2.1 below. The key fact about the matrix $A$ that implements this forward Euler propagation is that it is symmetric and "tree-tridiagonal" (also known as of "Hines" form (Hines, 1984)): all off-diagonal elements $A_{x w}$ are zero unless the compartments $x$ and $w$ are nearest neighbors on the dendritic tree.

Now the focus of this paper is the efficient implementation of the Kalman filter recursion (Durbin and Koopman, 2001) for computing the forward mean

$$
\mu_{t}=E\left(V_{t} \mid Y_{1: t}\right)
$$

and covariance

$$
C_{t}=\operatorname{Cov}\left(V_{t} \mid Y_{1: t}\right) \text {, }
$$

where $Y_{1: t}$ denotes all of the observed data $\left\{y_{s}\right\}$ up to time $t$. The Kalman recursions are ${ }^{4}$ :

$$
\begin{aligned}
C_{t} & =\left[\left(A C_{t-d t} A^{T}+\sigma^{2} d t I\right)^{-1}+B^{T} W^{-1} B\right]^{-1} \\
\mu_{t} & =C_{t}\left[\left(A C_{t-d t} A^{T}+\sigma^{2} d t I\right)^{-1}\left(A \mu_{t-d t}+i_{t} d t\right)+B^{T} W^{-1}\left(y_{t}-\mu_{t}^{y}\right)\right] .
\end{aligned}
$$

We have suppressed the possible time-dependence of $B$ and $W$ in the observation equation for notational clarity; the extension of these equations to the general case is standard (Durbin and Koopman, 2001). Note that the computation of the inverses in the recursion for $C_{t}$ requires $O\left(N^{3}\right)$ time in general, or $O\left(N^{2}\right)$ time (via the Woodbury lemma) if the observation matrix $B$ is of low rank. In either case, $O\left(N^{2}\right)$ space is required to store $C_{t}$.

These recursions are typically initialized with the marginal equilibrium covariance (i.e., the steady-state covariance of $V_{t}$ in the absence of any observations):

$$
C_{0}=\lim _{t \rightarrow \infty} \operatorname{Cov}\left(V_{t}\right)
$$

This equilibrium covariance $C_{0}$ satisfies the discrete Lyapunov equation (Brockwell and Davis, 1991)

$$
A C_{0} A^{T}+\sigma^{2} d t I=C_{0} .
$$

This equation can be solved explicitly here: applying the standard moving-average recursion (Brockwell and Davis, 1991) for the autoregressive model $V_{t}$ leads to

$$
C_{0}=\sum_{i=0}^{\infty} A^{i} \operatorname{cov}(\epsilon)\left(A^{T}\right)^{i}
$$

in general, and in our case, $\operatorname{cov}(\epsilon)=\sigma^{2} d t I$ and $A^{T}=A$, so this reduces to the explicit solution

$$
C_{0}=\sigma^{2} d t \sum_{i=0}^{\infty} A^{2 i}=\sigma^{2} d t\left(I-A^{2}\right)^{-1}
$$

\footnotetext{
${ }^{3}$ We will assume throughout this paper that the structural anatomy of the dendritic tree (i.e., the neighborhood structure $N(x)$ ) is fully known.

${ }^{4}$ For simplicity, we focus exclusively on the forward Kalman filter recursion in this paper, to compute $p\left(V_{t} \mid Y_{1: t}\right)$, but note that all of the methods discussed here can be applied to obtain the forward-backward (Kalman smoother) density $p\left(V_{t} \mid Y_{1: T}\right)$, for $t<T$.
} 

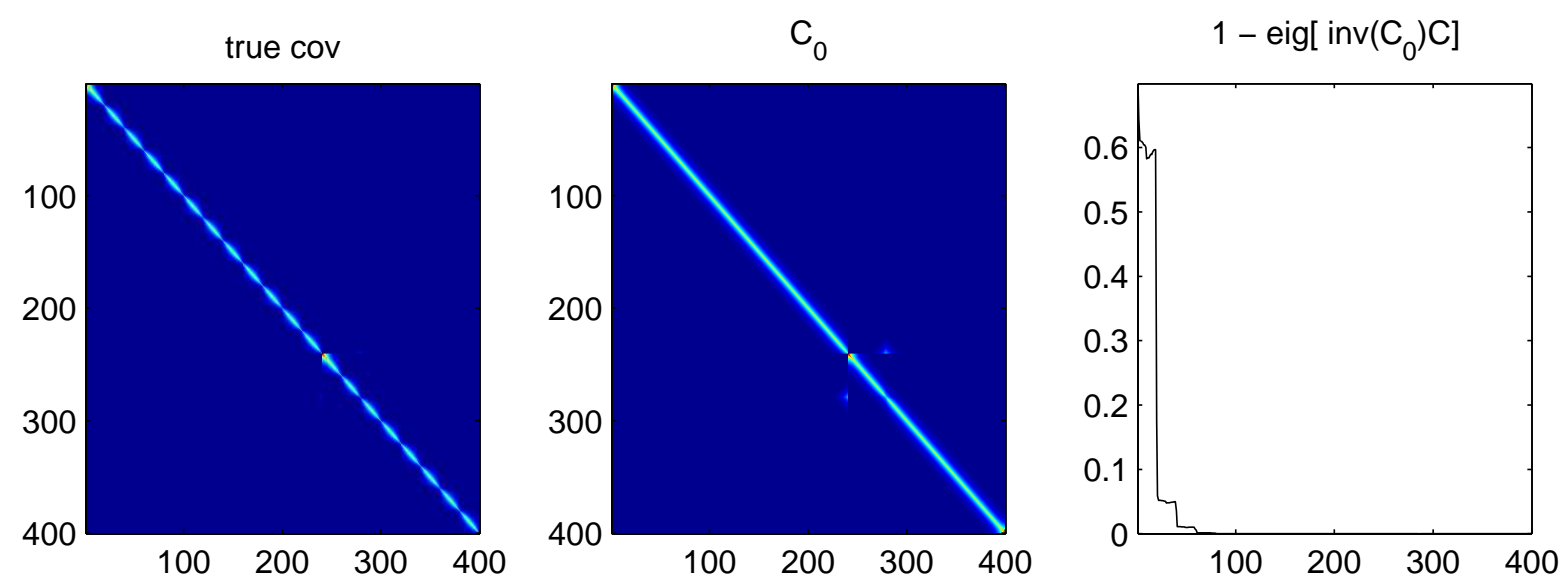

Figure 1: $C_{t}$ is fairly close to $C_{0}$; in particular, $I-C_{0}^{-1} C_{t}$ has low effective rank. $N=400$. $n=20$ : each 20-th compartment is observed. Observation noise set to be about $1 / 2$ of the marginal variance. This neuron had one branch; the branch point is visible at about compartment 230. Left: true $C_{t}$ ( $t$ is taken to be large enough that $C_{t}$ has converged to its limit, the solution of the corresponding Riccati equation (Durbin and Koopman, 2001)). Middle: $C_{0}$. Both $C_{0}$ and $C$ have been divided by $\max \left(C_{0}\right)$, to improve visibility. Right: spectrum of $I-C_{0}^{-1} C_{t}$. Note that the magnitude of the eigenvalues falls sharply after the 20-th eigenvalue, and again after the 40-th eigenvalue; an approximation of rank about 60 would seem to suffice for $I-C_{0}^{-1} C_{t}$ here.

whenever the dynamics matrix $A$ is stable (all eigenvalues have absolute value less than one); for the cable equation written in forward Euler form, $A$ may be guaranteed to be stable when the timestep $d t$ is sufficiently small. Note that we do not represent $C_{0}$ directly, as this would require $O\left(N^{2}\right)$ storage and computation time. If desired, we can compute the diagonal elements of $C_{0}$ (corresponding to the marginal equilibrium variances of the voltages at each compartment $x$ ) via the junction tree algorithm (Jordan, 1999; Weiss and Freeman, 2001; Shental et al., 2008), which is guaranteed to require just $O(N)$ time (and space) because the potential matrix $C_{0}^{-1} \propto\left(I-A^{2}\right)$ has a generalized tree structure: $\left[C_{0}^{-1}\right]_{x w}$ is nonzero only if comparments $x$ and $w$ are nearest- or second-nearest neighbors.

With these preliminaries out of the way, we can move on to the main contribution of this paper. The basic idea is that, in low-SNR conditions, $C_{t}$ should be close to $C_{0}$ : i.e., we should be able to represent the time-varying covariance $C_{t}$ as a small perturbation about the steadystate solution $C_{0}$, in some sense. This intuition is illustrated in Fig. 1 . If we compare $C_{t}$ to $C_{0}$ by computing the spectrum of $C_{0}^{-1} C_{t}$, we see that only a small fraction of the eigenvalues of $C_{0}^{-1} C_{t}$ are significantly different from one. Thus $C_{0}^{-1} C_{t}$ may be approximated as a low-rank perturbation of the identity matrix, or equivalently, $C_{t}$ may be approximated as a low-rank perturbation of $C_{0}$. The effective rank of this perturbation will depend on the SNR of the observations, specifically on the scale of the observation noise $W$ and on the dimensionality $n$ of the observation vector $y_{t}$ : the larger $n$ is, or the smaller $W$ is, the larger the effective rank of $I-C_{0}^{-1} C_{t}$.

Thus, more concretely, we will approximate $C_{t}$ as

$$
C_{t} \approx C_{0}+U_{t} D_{t} U_{t}^{T}
$$


where $U_{t} D_{t} U_{t}^{T}$ is a low-rank matrix we will update directly. We can compute and update the perturbations $U_{t}$ and $D_{t}$ in $O(N)$ time, as discussed in detail below. We should note that a great deal of related work on low-rank approximations to $C_{t}$ has appeared in the engineering and applied math literature (Verlaan, 1998; Treebushny and Madsen, 2005; Gillijns et al., 2006; Chandrasekar et al., 2008). However, we are not aware of previous low-rank approximations specialized to the case of low-SNR observations; note that, as discussed above, there is no good justification for approximating $C_{t}$ itself as low-rank in this setting.

We now derive the $O(N)$ low-rank updates for $\mu_{t}, U_{t}$, and $D_{t}$. First, write

$$
\begin{aligned}
\left(A C_{t-d t} A^{T}+\sigma^{2} d t I\right)^{-1} & =\left(A\left[C_{0}+U_{t-d t} D_{t-d t} U_{t-d t}^{T}\right] A^{T}+\sigma^{2} d t I\right)^{-1} \\
& =\left(C_{0}+A U_{t-d t} D_{t-d t} U_{t-d t}^{T} A^{T}\right)^{-1}
\end{aligned}
$$

the second equality follows from eq. (3). Now apply the Woodbury matrix lemma:

$$
\begin{aligned}
\left(C_{0}+A U_{t-d t} D_{t-d t} U_{t-d t}^{T} A^{T}\right)^{-1} & =C_{0}^{-1}-C_{0}^{-1} A U_{t-d t}\left(D_{t-d t}^{-1}+U_{t-d t}^{T} A^{T} C_{0}^{-1} A U_{t-d t}\right)^{-1} U_{t-d t}^{T} A^{T} C_{0}^{-1} \\
& =C_{0}^{-1}-R_{t} Q_{t} R_{t}^{T},
\end{aligned}
$$

where we have abbreviated

$$
R_{t}=C_{0}^{-1} A U_{t-d t}
$$

and

$$
Q_{t}=\left(D_{t-d t}^{-1}+U_{t-d t}^{T} A^{T} C_{0}^{-1} A U_{t-d t}\right)^{-1} .
$$

Now plug this into the covariance update:

$$
\begin{aligned}
C_{t} & =\left[\left(A C_{t-d t} A^{T}+\sigma^{2} d t I\right)^{-1}+B^{T} W^{-1} B\right]^{-1} \\
& =\left[C_{0}^{-1}-R_{t} Q_{t} R_{t}^{T}+B^{T} W^{-1} B\right]^{-1}
\end{aligned}
$$

We see that the update is of low-rank form. To apply Woodbury again, we just need to simplify the term $-R_{t} Q_{t} R_{t}^{T}+B^{T} W^{-1} B$. Choose an orthogonal basis

$$
O_{t}=\operatorname{orth}\left(\left[\begin{array}{ll}
R_{t} & B
\end{array}\right]\right)
$$

and then write

$$
-R_{t} Q_{t} R_{t}^{T}+B^{T} W^{-1} B=O_{t} M_{t} O_{t}^{T}
$$

with

$$
M_{t}=-O_{t}^{T} R_{t} Q_{t} R_{t}^{T} O_{t}+O_{t}^{T} B^{T} W^{-1} B O_{t} .
$$

Now, finally, apply Woodbury again ${ }^{5}$ :

$$
\begin{aligned}
C_{t} & =\left[C_{0}^{-1}-R_{t} Q_{t} R_{t}^{T}+B^{T} W^{-1} B\right]^{-1} \\
& =\left[C_{0}^{-1}+O_{t} M_{t} O_{t}^{T}\right]^{-1} \\
& =C_{0}-C_{0} O_{t}\left(M_{t}^{-1}+O_{t}^{T} C_{0} O_{t}\right)^{-1} O_{t}^{T} C_{0} .
\end{aligned}
$$

\footnotetext{
${ }^{5}$ It is well-known that the Woodbury formula can be numerically unstable when the observation covariance $W$ is small (i.e., the high-SNR case). Our applications here concern the low-SNR case instead, and therefore we have not had trouble with these numerical instabilities. However, it should be straightforward to derive a low-rank square-root filter (Howard and Jebara, 2005; Treebushny and Madsen, 2005; Chandrasekar et al., 2008) to improve the numerical stability here, if needed.
} 

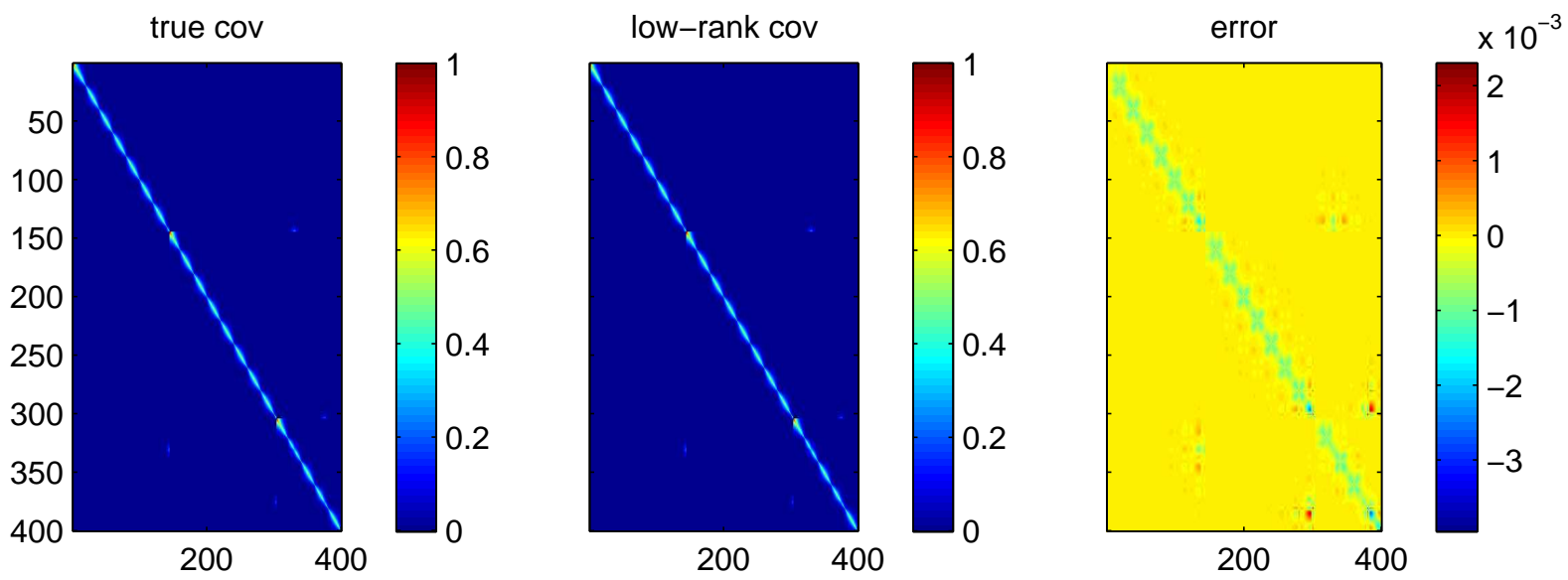

Figure 2: $C_{t}$ is very well-approximated by $C_{0}+U_{t} D_{t} U_{t}^{T} ; \operatorname{rank}(U)=39$ here (chosen automatically by the algorithm using $c=.999$ in equation (6)). This tree has two branches (near compartments 150 and 300); otherwise simulation details are as in Fig. 1. $U$ and $D$ denote the large- $t$ limits of $U_{t}$ and $D_{t}$, respectively. Left: true $C_{t}$. Middle: $C_{0}+U D U^{T}$. Right: error of the approximation, $C_{t}-\left(C_{0}+U D U^{T}\right)$. As in Fig. 2, each panel has been divided by $\max \left(C_{0}\right)$, to improve visibility.

We obtain $U_{t}$ and $D_{t}$ by truncating the SVD of the expression on the right-hand side: in Matlab, for example, do

$$
\left[U^{\prime}, D^{\prime}\right]=\operatorname{svd}\left(C_{0} O_{t}\left(M_{t}^{-1}+O_{t}^{T} C_{0} O_{t}\right)^{-1 / 2},{ }^{'} \text { econ }^{\prime}\right),
$$

then choose $U_{t}$ as the first $k$ columns of $U^{\prime}$ and $D_{t}$ as the negative square of the first $k$ diagonal elements $D^{\prime}$, where $k$ is chosen to be large enough (for accuracy) and small enough (for computational tractability). We have found that a reasonable choice of $k$ is as the least solution of the inequality:

$$
\sum_{i \leq k} D_{i i}^{2} \geq c \sum_{i} D_{i i}^{2}
$$

i.e., choose $k$ to capture at least a large fraction $c$ of the variance in the right-hand term perturbing $C_{0}$ in equation (5). Figure 2 illustrates the accuracy of this approximation for the full $C_{t}$.

Now the update for $\mu_{t}$ is relatively standard:

$$
\begin{aligned}
\mu_{t} & =C_{t}\left[\left(A C_{t-d t} A^{T}+\sigma^{2} d t I\right)^{-1} m_{t}+B^{T} W^{-1}\left(y_{t}-\mu_{t}^{y}\right)\right] \\
& =\left(P_{t}^{-1}+B^{T} W^{-1} B\right)^{-1}\left[P_{t}^{-1} m_{t}+B^{T} W^{-1}\left(y_{t}-\mu_{t}^{y}\right)\right] \\
& =\left(P_{t}-P_{t} B^{T}\left(W+B P_{t} B^{T}\right)^{-1} B P_{t}\right)\left[P_{t}^{-1} m_{t}+B^{T} W^{-1}\left(y_{t}-\mu_{t}^{y}\right)\right] \\
& =m_{t}-P_{t} B^{T}\left(W+B P_{t} B^{T}\right)^{-1} B\left[s_{t}+m_{t}\right]+s_{t},
\end{aligned}
$$

where we have made the abbreviations

$$
\begin{gathered}
P_{t}=C_{0}+A U_{t-d t} D_{t-d t} U_{t-d t}^{T} A^{T}, \\
m_{t}=A \mu_{t-d t}+i_{t} d t,
\end{gathered}
$$



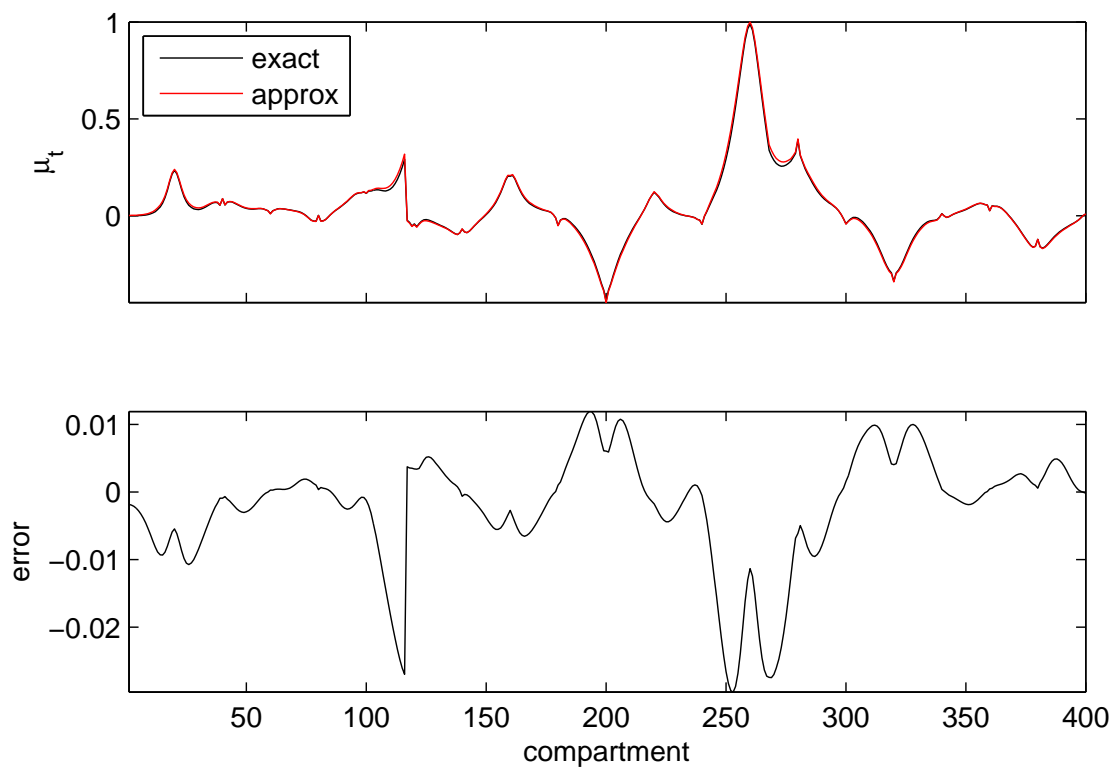

Figure 3: The low-rank approximation for $\mu_{t}$ is similarly accurate. Top: true $\mu_{t}$ (black) and approximate $\mu_{t}$ (red) at an arbitrarily chosen timepoint $t$ (the approximation quality remains roughly constant over the course of the trial; data not shown). Bottom: error of the approximation, $\mu_{t}-\mu_{t}^{\text {approx }}$. As in Fig. 2, each panel has been divided by $\max \left(\left|\mu_{t}\right|\right)$, to improve visibility. Note that the error is small; the details of $\mu_{t}$ here are driven by noise and are relatively unimportant in this example. All parameters of the simulation are as in Fig. 2.

and

$$
s_{t}=P_{t} B^{T} W^{-1}\left(y_{t}-\mu_{t}^{y}\right) .
$$

Note that we update the mean $\mu_{t}$ first, then truncate $U_{t}$ and $D_{t}$. See Fig. 3 for an illustration.

To sum up, the key point is that all of the necessary computations described above can be done in $O(N)$ time and memory. $C_{0}$ need never be computed explicitly; instead, all we need is to multiply and divide by $C_{0}^{-1}$ (by "divide," we mean to solve equations of the form $C_{0}^{-1} v=r$ for the unknown vector $v$ and known vector $r$ ); the former can be done directly with a sparse matrix multiply, and the latter by the junction tree algorithm, or with, e.g., $C_{0}^{-1} \backslash r$ in Matlab (where $C_{0}^{-1}$ is represented as a sparse matrix), which has efficient elimination tree code built in to its sparse matrix solver. The posterior marginal variance $\left[C_{t}\right]_{i i}$ can also easily be computed in $O(N)$ time, to provide errorbars around $\mu_{t}$ : given the diagonal of $C_{0}$ (obtained via the junction tree algorithm, as discussed above), computing the diagonal of $C_{t}$ just requires us to sum the squared elements of $\left(-D_{t}\right)^{1 / 2} U_{t}$.

We should also note that the method can be sped up significantly in the special case that $B$ is time-invariant: in this case, $C_{t}$ will converge to a limit (as an approximate solution of the corresonding Riccati equation), and we can stop recomputing $U_{t}$ and $D_{t}$ on every time step. More generally, if $B_{t}$ is time-varying in a periodic manner (e.g., we are repeatedly sequentially scanning over a region of dendrite in an imaging experiment), then $C_{t}$ will also be periodic, and we can store a period's worth of $U_{t}$ and $D_{t}$ in memory, instead of continuing to recompute these on each timestep. 


\subsection{Incorporating implicit methods for solving the cable equation}

It is well-known that the forward Euler method described above for solving the cable equation is unstable for large values of $a d t$ (where $a$ denotes the intercompartmental conductance) (Hines, 1984; Press et al., 1992). The following "backwards Euler" (implicit) discretization is unconditionally stable and can therefore be made much faster, by choosing a larger timestep $d t^{6}$ :

$$
V_{t+d t}(x)=V_{t}(x)+d t\left(-g_{x} V_{t+d t}(x)+\sum_{w \in N(x)} a_{x w}\left[V_{t+d t}(w)-V_{t+d t}(x)\right]\right) .
$$

We can write this in matrix-vector form as

$$
V_{t+d t}=V_{t}+R V_{t+d t}
$$

for a suitable Hines matrix $R$, or in other words

$$
V_{t+d t}=(I-R)^{-1} V_{t}
$$

It is conceptually straightforward to replace $A$ with $(I-R)^{-1}$ in our above derivations. Of course, $(I-R)^{-1}$ is not a sparse matrix, so we do not want to make this substitution explicitly; instead, we will use our efficient matrix-divide methods to solve for any term of the form $(I-R)^{-1} v$. In particular, we need to multiply and divide by $C_{0}$. We have

$$
C_{0}^{-1}=\frac{1}{\sigma^{2} d t}\left[I-A^{2}\right]=\frac{1}{\sigma^{2} d t}\left[I-(I-R)^{-2}\right] ;
$$

$(I-R)^{2}$ has tree-bandwidth two (i.e., matrix elements corresponding to pairs of compartments which are further than two nodes apart on the tree are zero), so we can efficiently divide by this matrix. Similarly,

$$
\begin{aligned}
C_{0} & =\sigma^{2} d t\left[I-A^{2}\right]^{-1} \\
& =\sigma^{2} d t\left[I-(I-R)^{-2}\right]^{-1} \\
& =\sigma^{2} d t\left[(I-R)^{2}-I\right]^{-1}(I-R)^{2}
\end{aligned}
$$

multiplying by $(I-R)^{2}$ is clearly fast, and since $\left[(I-R)^{2}-I\right]$ has tree-bandwidth two, we can efficiently divide by this matrix as well.

Second-order semi-implicit Crank-Nicholson methods (Hines, 1984; Press et al., 1992) may be incorporated in a similar fashion, though we have found the fully implicit method described here to be adequate for our needs, and have not pursued the Crank-Nicholson implementation in depth.

At this point it is worth pausing to summarize our approach. Given the reconstructed neuron file (containing the neighborhood structure $N(x)$ ) and the observed matrix of voltagesensitive measurements $Y$, the first step is to define the parameters in the dynamics and observations equations (1) and (2). Specifically, we must form the dynamics matrix $A$, the input current vector $i_{t}$, the observations matrix $B_{t}$, and the noise parameters $\sigma^{2}, W_{t}$, and

\footnotetext{
${ }^{6}$ We have suppressed the input current $i_{t}(x)$ and noise $\epsilon_{t}(x)$ in the following, for notational simplicity; inclusion of the input current term does not significantly complicate the derivations in this section, since the steady-state covariance $C_{0}$ does not depend on $i_{t}(x)$.
} 
$\mu_{t}^{y}$. The observation parameters $B_{t}, W_{t}$, and $\mu_{t}^{y}$ are characteristics of the imaging apparatus, and good initial estimates of these parameters should therefore be available directly: as discussed above, the $i$-th row of $B_{t}$ specifies how the observed voltage at each compartment is weighted and summed to form the observations at the $i$-th pixel at time $t$, while

$W_{t}$, and $\mu_{t}^{y}$ specify the noise covariance and bias of these observations, respectively. The dynamics parameters $A, i_{t}$, and $\sigma$ can also be initialized in a straightforward manner: in the simplest case, we could let the intercompartmental couplings $a_{x w}$ and membrane conductance $g_{x}$ be proportional to the compartment diameter, reducing the difficult task of choosing $A$ to the much simpler job of selecting two proportionality coefficients. As discussed in (Huys and Paninski, 2009), these parameters (along with the dynamics noise variance $\sigma^{2}$ ) may be estimated using an iterative expectation-maximization approach, and initialized using prior knowledge from previous estimates in similar neurons. Once all of these system parameters are chosen, we need only select the fraction of variance parameter $c$ (from (6)): larger values of $c$ lead to greater accuracy at the cost of speed. Then we simply iterate the update equations above to obtain the desired filter outputs $E\left(V_{t} \mid Y_{1: t}\right)$ and $\operatorname{Cov}\left(V_{t} \mid Y_{1: t}\right)$. Sample code implementing the simulations described in the next section is available at http://www.stat.columbia.edu/ liam/research/abstracts/dendrite-kalman-abs.html.

\section{Applications to spatially-subsampled dendritic trees}

We illustrate the fast implicit Kalman filtering methods described in the last section with two simulations here. The results of the simulations are best viewed in movie form (lowrank-speckle.mp4 and low-rank-horiz.mp4); we show snapshots and two-dimensional representations of these movies in Figs. 4-8. In the first three figures, we demonstrate the output of the fast Kalman filter given sparsely-sampled spatial observations of a complex, real dendritic tree (reconstructed from a rabbit starburst amacrine cell), and in the last two figures we apply the fast Kalman filter to highly spatially-coarsened observations: we observe only the vertically-summed, noise-corrupted voltage on the tree, instead of retaining full spatial information about the observations $Y$. (This latter simulation is an effort to roughly emulate the results of fast planar imaging methods, as discussed for example in (Holekamp et al., 2008).)

In each case, the filter is able to recover the spatiotemporal voltage fairly well (and is even able to restore some of the spatial information that is apparently lost by vertically summing the observations in Figs. 7-8), though the filtered voltage $E\left(V_{t} \mid Y_{1: t}\right)$ consistently underestimates and smooths the true voltage $V_{t}$, as we would expect of any optimal linear filter. We emphasize that our goal here is to give a qualitative picture of the filter's behavior, to illustrate that the approximate methods developed above do not lead to any visible artifacts in the filter output; for more quantitative analyses of the estimation performance of the Kalman and other Bayesian filters applied in this setting (albeit in the context of much lowerdimensional neural models, where more direct computational approaches are tractable), see (Huys and Paninski, 2009). 

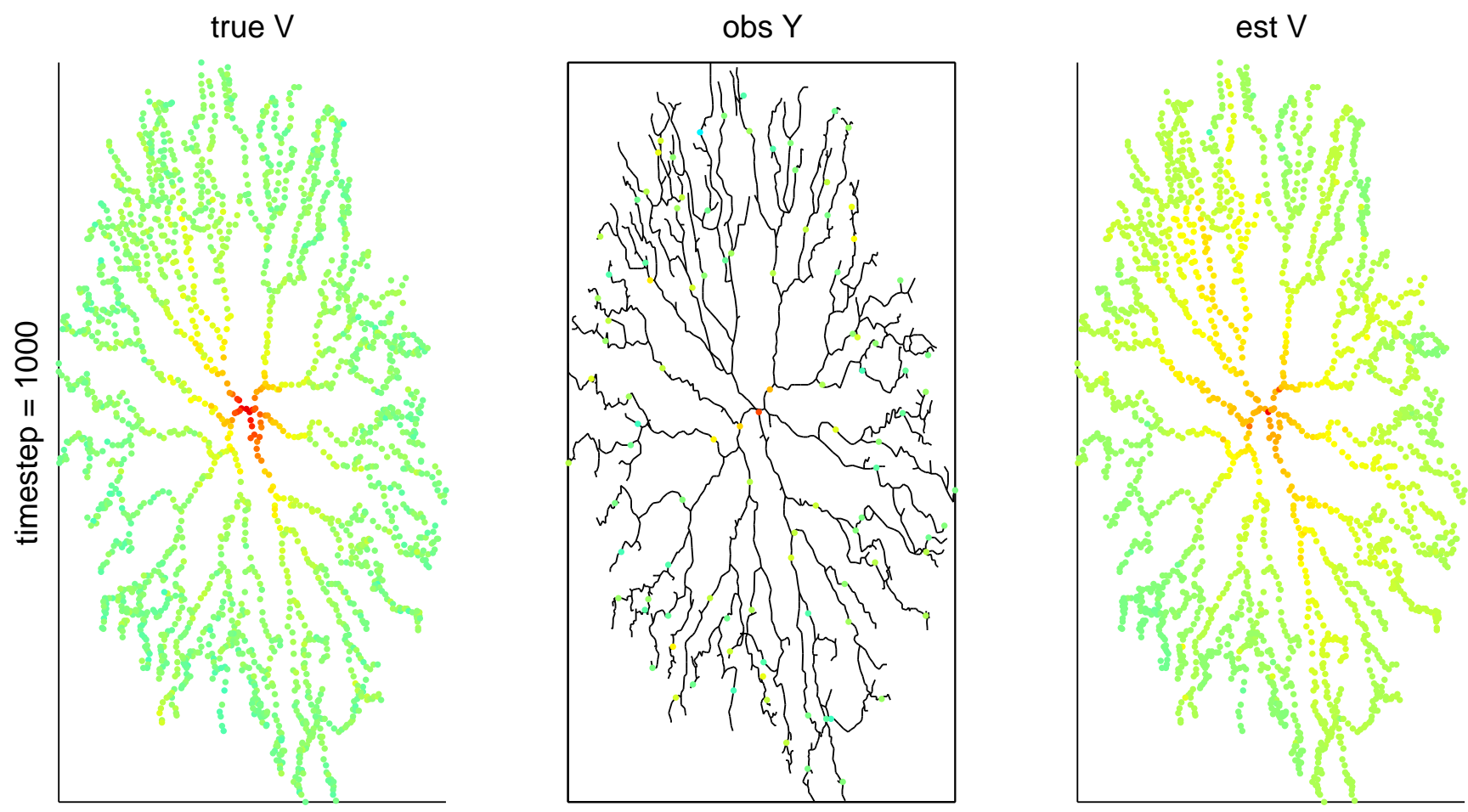

Figure 4: Snapshot of a simulation in which we made noisy observations of just 100 out of $>2000$ compartments. The full simulation may be viewed in movie form in low-rankspeckle.mp4. The neuronal geometry is taken from the "THINSTAR" file (rabbit starburst amacrine cell) available at http://neuromorpho.org; see (Bloomfield and Miller, 1986) for details. Left panel: true voltage $V_{t}(x)$ at timestep $t=1000$; color indicates voltage (red corresponds to high voltage and blue corresponds to low). Each dot represents an individual compartment. The somatic compartment (center, red) was driven with a sinusoidal input (see Fig. 5 below for details). Middle panel: observed data $y_{t} .100$ compartments were randomly chosen and then observed (with noise) at each time step. Underlying black trace indicates structure of dendritic tree. Each colored dot indicates an observed compartment. Right panel: filter estimate $E\left(V_{t}(x) \mid Y_{1: t}\right)$. The first two panels are plotted on the same color scale; the third is plotted on its own color scale for improved visibility. See Fig. 5 for the full $V_{t}(x), y_{t}$, and $E\left(V_{t}(x) \mid Y_{1: t}\right)$ for all times $t$. 

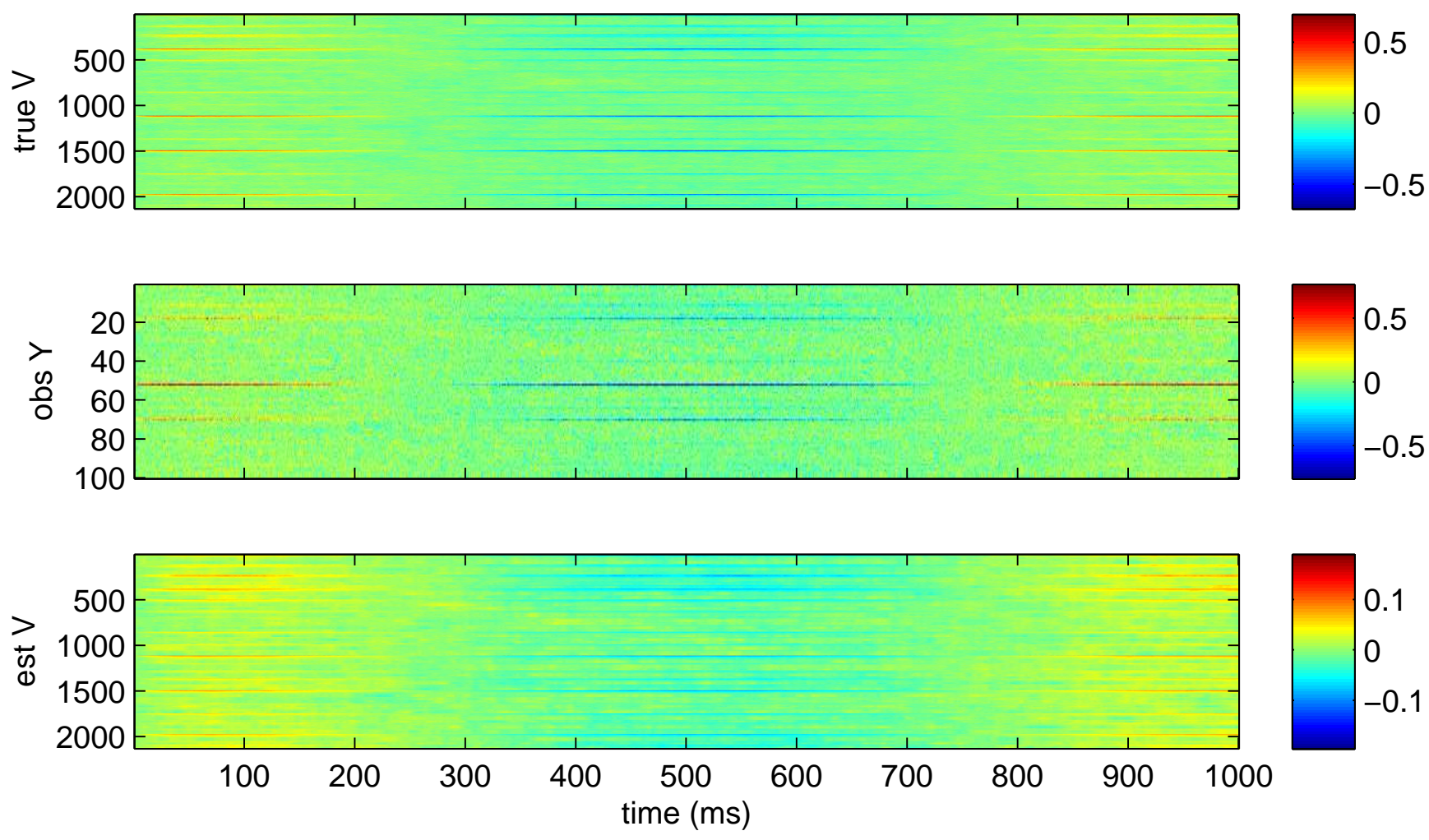

Figure 5: Full spatiotemporal voltage traces corresponding to the snapshots shown in Fig. 4; data matrices may be obtained by running the code at http://www.stat.columbia.edu/ liam/research/abstracts/dendrite-kalman-abs.html. Top: true spatiotemporal voltage $V_{t}(x)$; middle: observed data $y_{t}$; bottom: filtered voltage $E\left(V_{t}(x) \mid Y_{1: t}\right)$. All plots share the same units (voltage scaled by a factor which in this case may be ignored), but are plotted with different color scaling, for improved visibility. In this simulation, a $1 \mathrm{~Hz}$ sinusoidal current was injected into compartment 1 (visible as the soma, colored red in the left panel in Fig. 4); however, this current was not considered known (i.e., $i_{t}$ in eq. (1) was set to zero), to make the filtering problem a bit more challenging here. The average SNR per compartment (computed by taking the average signal variance $\operatorname{var}\left(V_{t}(x)\right)$ over all the compartments and dividing by the observation noise variance, normalized by the fraction of observed compartments, $n / N)$ was about $4 \%$. Note that the filtered voltage $E\left(V_{t} \mid Y_{1: t}\right)$ underestimates and smooths the true voltage $V$, both spatially and temporally. 

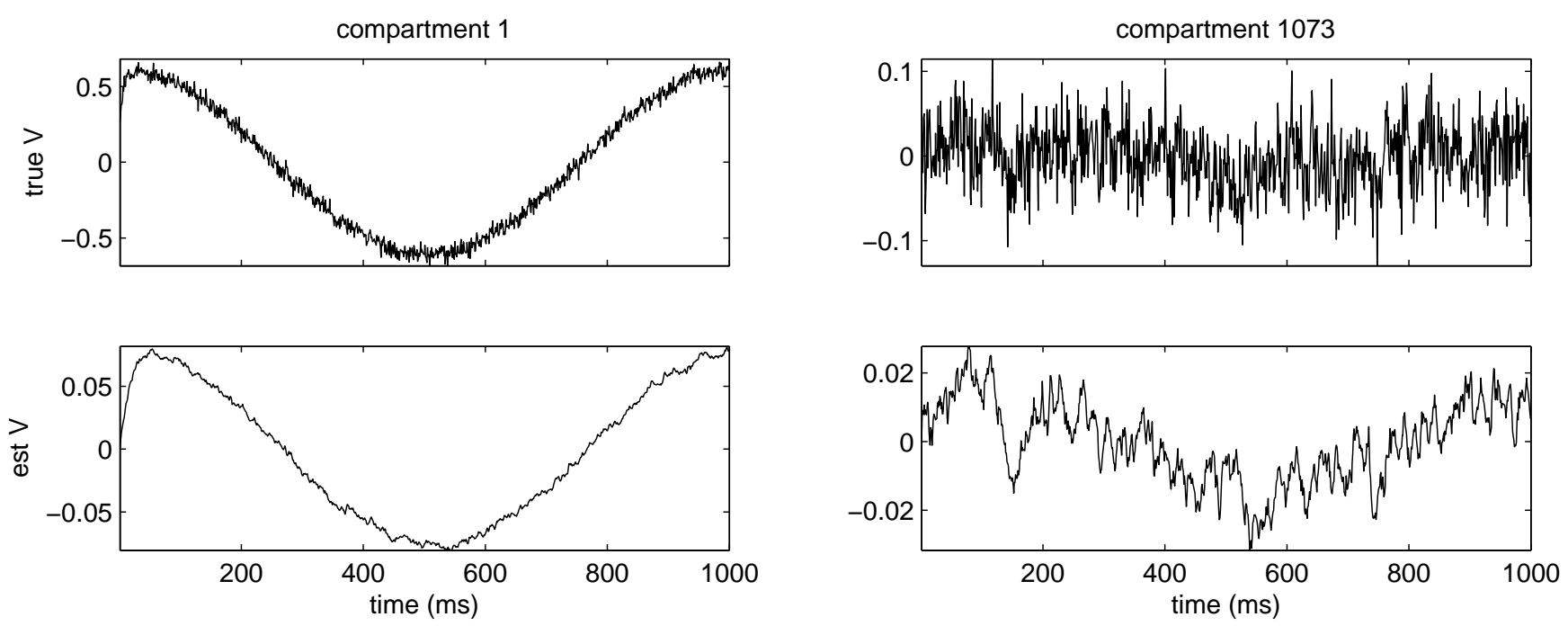

Figure 6: Example one-dimensional voltage traces selected from the data matrices shown in Fig. 5. The true and inferred somatic voltages are shown on the left (compartment 1); voltages from a randomly-chosen compartment are shown on the right. Neither of these compartments were among the subset of 100 compartments which were observed directly in the middle panels of Figs. 4-5. The effect of the sinusoidal somatic input current is most strongly visible on the left, and is more attenuated in the distal compartment shown on the right. Again, note that the filtered voltage significantly underestimates and smooths the true voltage $V$, due to the low effective SNR here. 

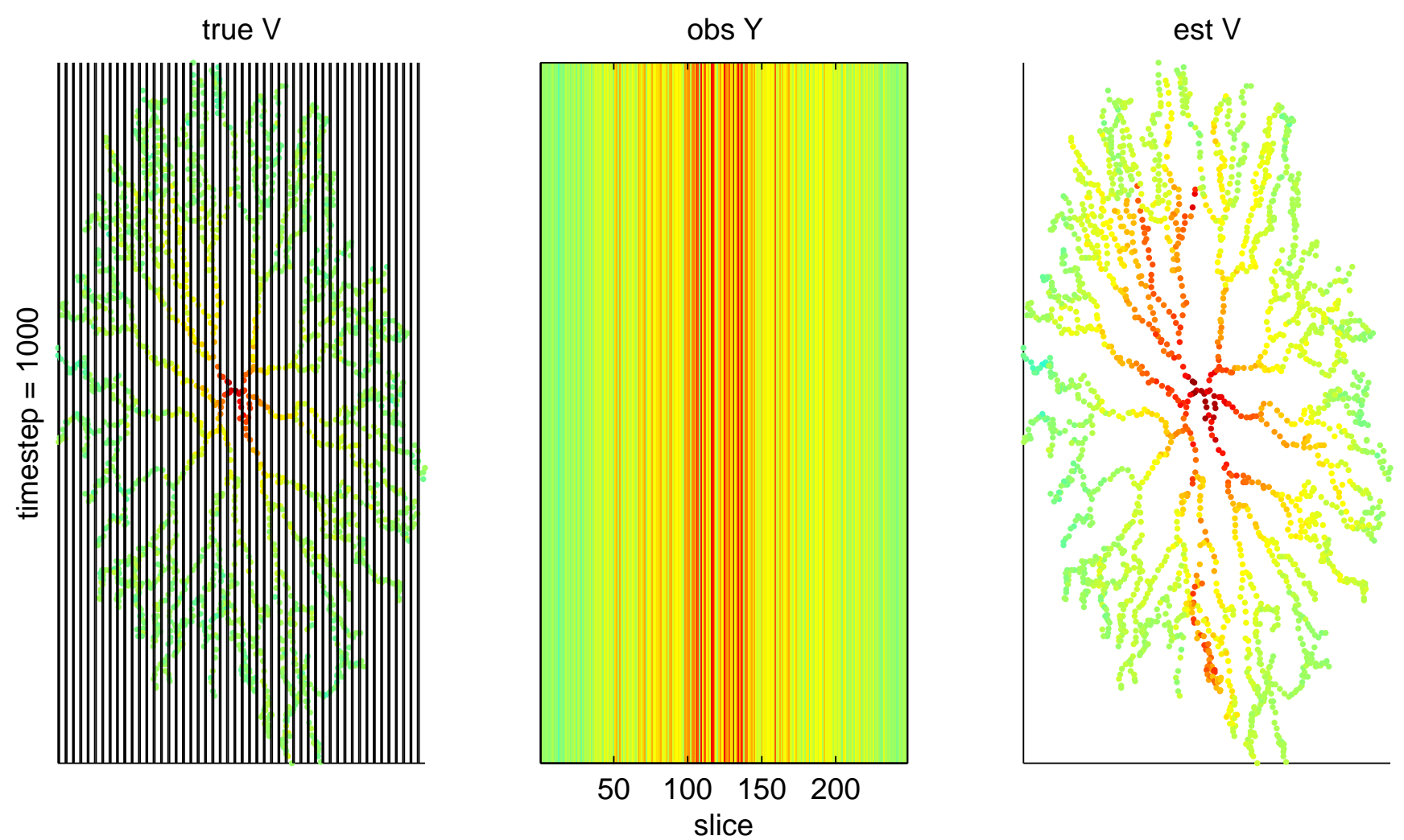

Figure 7: Snapshot of a simulation similar to that shown in Fig. 4-5, but with verticallyscanned observations (i.e., the dynamics equation is identical here, but the observation equation is different). Layout and conventions are as in Fig. 4. In this case, the observations $Y$ were formed by summing the observed voltages over the 250 nonoverlapping "slices" shown in the left panel (only every fifth slice is shown, for visibility) and adding noise. The full simulation may be viewed in movie form in low-rank-horiz.mp4. 

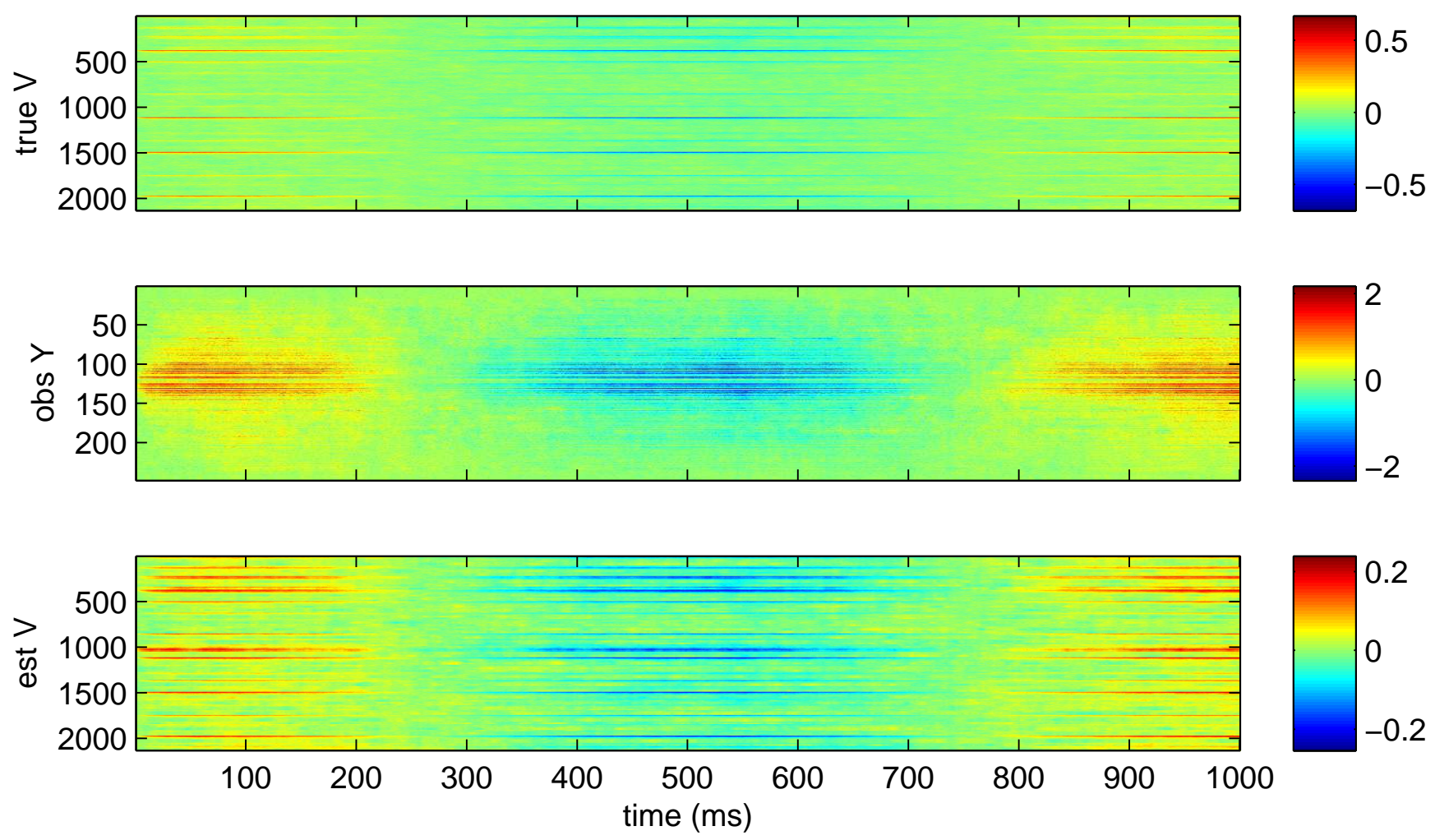

Figure 8: Full spatiotemporal voltage traces corresponding to the snapshots shown in Fig. 7. As in Figs. 4-5, sinusoidal current was injected into the soma but not included in the Kalman filter $\left(i_{t}=0\right)$. Again, the filtered voltage $E\left(V_{t} \mid Y_{1: t}\right)$ significantly smooths and shrinks the true voltage $V$. In addition, the vertical sampling here leads to significant loss of spatial detail relative to the simulation shown in Figs. 4-5. 


\section{Extending the basic method}

In this section we discuss several important extensions of the basic methods introduced in section 2 .

\subsection{Temporally-filtered observations and dynamics noise}

First we would like to relax the assumption that the observations $y_{t}$ are instantaneously related to the voltage $V_{t}$. For example, it is well-known that whole-cell recordings introduce filtering effects, due to the RC dynamics of the electrode-cell system (Brette et al., 2007); similarly, some voltage sensors (particularly genetically-encoded voltage sensors) respond noninstantaneously to voltage steps and thus effectively lowpass filter the voltage (Knopfel et al., 2006).

We therefore modify our basic dynamics and observation equations to incorporate this temporal filtering of the observations. For clarity, we will treat just the simplest case, in which the observations are filtered in a first-order (low-pass) manner, though generalizations are straightforward. We write

$$
\begin{aligned}
q_{t+d t} & =A q_{t}+\sqrt{d t} \epsilon_{t}, \epsilon_{t} \sim \mathcal{N}(0, I) \\
z_{t+d t}^{i} & =z_{t}^{i}+\left(a q_{t}^{i}-\frac{z_{t}^{i}}{\tau}\right) d t+\sqrt{d t} \xi_{t}^{i}, \quad \xi_{t}^{i} \sim \mathcal{N}\left(0, \sigma^{2}\right) \\
y_{t} & =B z_{t}+\eta_{t}, \eta_{t} \sim \mathcal{N}(0, W) .
\end{aligned}
$$

Here we have introduced a scaled voltage variable $q_{t}$ to simplify the notation below, and we make linear observations of the low-pass filtered variable $z_{t} ; \tau$ denotes the time constant of this filter, and $a$ denotes the filter gain. (A minor technical point: since we do not observe $q_{t}$ directly, there is a free scale parameter which must be fixed here; thus we have set the $q$-dynamics noise variance to 1 , and set the $z$-dynamics noise variance to $\sigma^{2}$. We have also set the observation mean $\mu_{t}^{y}$ to zero, and suppressed the input current $i_{t}(x)$ for notational simplicity; inclusion of these terms does not significantly complicate the following derivations, since again the steady-state covariance $C_{0}$ does not depend on $\mu_{t}^{y}$ or $i_{t}(x)$.)

Now the key to the basic methods described in section 2 is that we could compute the steady-state covariance $C_{0}$ of the state vector $q_{t}$ quite explicitly. In the case of interest here, the state vector is $\left(q_{t}, z_{t}\right)$; unfortunately, computing the steady-state covariance (which we will continue to denote as $C_{0}$ ) is a bit less trivial now, since the dynamics matrix is asymmetric, and therefore the infinite sum (4) is more difficult to compute. We write

$$
C_{0}=\left(\begin{array}{cc}
c_{0} & J \\
J^{T} & F
\end{array}\right)
$$

where we have abbreviated the upper-left block as

$$
c_{0}=\left(I+A^{2}\right)^{-1} d t
$$

(i.e., $c_{0}$ denotes the familiar steady-state covariance of $q_{t}$ ).

To compute the matrices $J$ and $F$, we rewrite the $z_{t}$ dynamics slightly,

$$
z_{t+d t}=b z_{t}+d q_{t}+\sqrt{d t} \xi_{t}, \xi_{t} \sim \mathcal{N}\left(0, \sigma^{2} I\right),
$$


where we have abbreviated $b=1-d t / \tau$ and $d=a d t$. Now we can write a simple recursion for the cross-covariance:

$$
\begin{aligned}
E\left(q_{t+d t} z_{t+d t}^{T}\right) & =E\left(\left[A q_{t}+\sqrt{d t} \epsilon_{t}\right]\left[b z_{t}+d q_{t}+\sqrt{d t} \xi_{t}\right]^{T}\right) \\
& =d A E\left(q_{t} q_{t}^{T}\right)+b A E\left(q_{t} z_{t}^{T}\right) .
\end{aligned}
$$

Here $E\left(q_{t} q_{t}^{T}\right)$ tends to $c_{0}$, as before, and $E\left(q_{t+d t} z_{t+d t}^{T}\right)$ should be equal to $E\left(q_{t} z_{t}^{T}\right)$ in the limit $t \rightarrow \infty$. This leads to the equation

$$
J=d(I-b A)^{-1} A c_{0} .
$$

Note that $J$ is symmetric, since it is a product of symmetric commuting terms.

We may derive a similar recursion for the covariance of $z_{t}$ :

$$
\operatorname{cov}\left(z_{t+d t}\right)=b^{2} \operatorname{cov}\left(z_{t}\right)+d^{2} \operatorname{cov}\left(q_{t}\right)+2 b d E\left(q_{t} z_{t}^{T}\right)+\sigma^{2} I d t .
$$

Taking limits as above, we compute

$$
F=\left(1-b^{2}\right)^{-1}\left(d^{2} c_{0}+2 b d J+\sigma^{2} I d t\right) .
$$

Now we need to efficiently multiply and divide by $C_{0}$. Multiplying by $C_{0}$ can clearly be done in $O(N)$ time. To divide, we write the inverse as

$$
C_{0}^{-1}=\left(\begin{array}{cc}
c_{0}^{-1}+c_{0}^{-1} J G^{-1} J c_{0}^{-1} & -G^{-1} J c_{0}^{-1} \\
-G^{-1} J c_{0}^{-1} & G^{-1}
\end{array}\right)
$$

where we have abbreviated the Schur complement

$$
G=F-J c_{0}^{-1} J
$$

Multiplying by $J$ and $c_{0}^{-1}$ can clearly be done in $O(N)$ time. After some cancellations, $G$ may be written as

$$
G=\left[\left(1-b^{2}\right)^{-1}\left(d^{2}\left(I-b^{2} A^{2}\right)+\sigma^{2} d t(I-b A)^{2} c_{0}^{-1}\right)-d^{2} A^{2}\right] c_{0}(I-b A)^{-2} .
$$

The term in brackets has a tree-bandwidth of 4 , and we can therefore divide by $G$ in $O(N)$ time, as desired. See Fig. 9 for an illustration.

A very similar approach may be used to handle the case of temporally-correlated input noise, e.g., synaptically-filtered noise arising from spontaneous release of neurotransmitter, followed by postsynaptic conductance transients which do not decay instantaneously. As above, we treat the case of a first-order lowpass filter, and begin with the recursion

$$
\begin{aligned}
q_{t+d t} & =A q_{t}+d w_{t}+\sqrt{d t} \epsilon_{t}, \epsilon_{t} \sim \mathcal{N}(0, I) \\
w_{t+d t} & =b w_{t}+\sqrt{d t} \xi_{t}, \xi_{t} \sim \mathcal{N}\left(0, \sigma^{2} I\right),
\end{aligned}
$$

where $w_{t}$ represents the filtered noise source. From here we may derive a simple recursion for the cross-covariance:

$$
\begin{aligned}
E\left(q_{t+d t} w_{t+d t}^{T}\right) & =E\left(\left[A q_{t}+d w_{t}+\sqrt{d t} \epsilon_{t}\right]\left[b w_{t}+\sqrt{d t} \xi_{t}\right]^{T}\right) \\
& =b A E\left(q_{t} w_{t}^{T}\right)+b d E\left(w_{t} w_{t}^{T}\right) .
\end{aligned}
$$


true $\mathrm{V}$
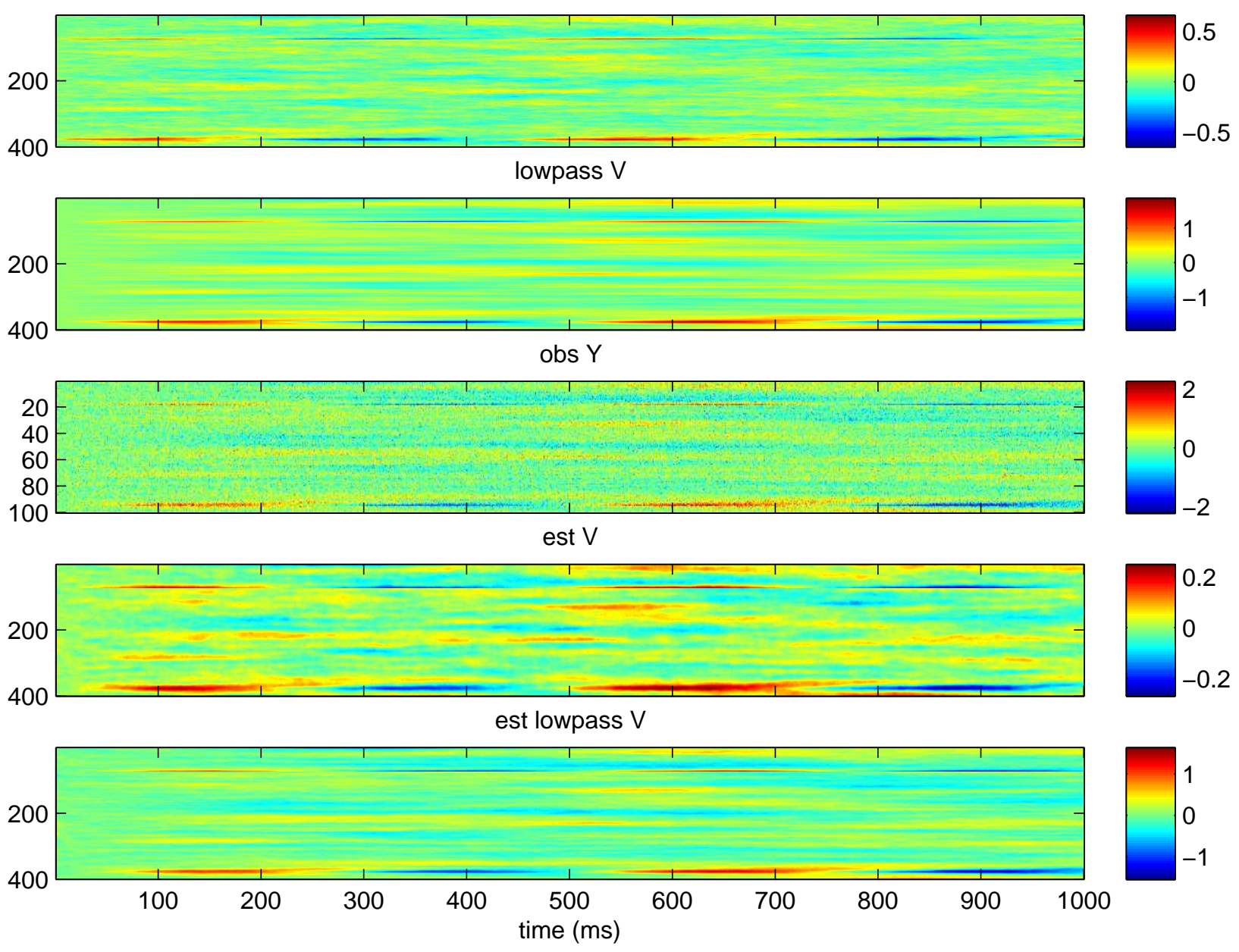

Figure 9: An example of the Kalman filter applied to lowpass-filtered voltage observations. Top panel: true spatiotemporal voltage. Sinusoidal current $(2 \mathrm{~Hz})$ was injected into a branch point of the tree (compartment 380); the tree was a simple one-branched structure with 400 compartments in this case. Second panel: lowpass-filtered $(\tau=100 \mathrm{~ms})$ voltage. Third panel: observed $Y$; the lowpass-filtered voltage was spatially subsampled $4 x$ and corrupted with noise. Fourth and fifth panels: output of Kalman filter: estimated voltage $V$ and lowpassfiltered voltage, respectively. Note that the Kalman filter is causal, so the estimated voltage $V$ lags the true $V$; to obtain a non-lagged estimate, we must run the full forward-backward Kalman smoother (data not shown).

Computing the steady-state solution of this recursion, we find that the cross-covariance $E\left(q_{t} w_{t}^{T}\right)$ tends to

$$
J=b d \sigma^{2}\left(1-b^{2}\right)^{-1}(I-b A)^{-1} .
$$

We also have that

$$
E\left(q_{t+d t} q_{t+d t}^{T}\right)=A E\left(q_{t} q_{t}^{T}\right) A^{T}+d^{2} E\left(w_{t} w_{t}^{T}\right)+2 d A E\left(q_{t} w_{t}^{T}\right)+I d t
$$


from which we conclude that the covariance $E\left(q_{t} q_{t}^{T}\right)$ tends to

$$
F=\left(I-A^{2}\right)^{-1}\left[\left(d^{2} \sigma^{2}\left(1-b^{2}\right)^{-1}+d t\right) I+2 b d^{2} \sigma^{2}\left(1-b^{2}\right)^{-1} A(I-b A)^{-1}\right] .
$$

The full steady-state covariance

$$
C_{0}=\left(\begin{array}{cc}
\left(1-b^{2}\right)^{-1} \sigma^{2} I & J \\
J & F
\end{array}\right)
$$

may now be multiplied and divided in $O(N)$ time using sparse Schur methods nearly identical to those described above.

\subsection{Inhomogeneous cable noise and quasi-active membranes}

In the case that the dynamics noise covariance $\operatorname{cov}(\epsilon)$ is diagonal but not proportional to the identity, the infinite sum (4) is even more difficult to compute (since $\operatorname{cov}(\epsilon)$ and $A$ will not commute in general). Standard direct methods for solving the Lyapunov equation (e.g., the Bartels-Stewart algorithm (Antoulas, 2005)) require an orthogonalization step that takes $O\left(N^{3}\right)$ time in general. There is a large applied mathematics literature on the approximate solution of Lyapunov equations with sparse dynamics (see e.g. (Sabino, 2007) for a nice review), but the focus of this literature is on the case that the noise covariance matrix is of low rank. Since we expect the voltage at every compartment to be perturbed by at least some noise (implying that the rank of the noise covariance is at least as large as $N$ ), these low-rank methods are less attractive here. We use a direct iterative method for computing $C_{0}$ instead.

Abbreviate $D=\operatorname{cov}(\epsilon)$. Now we recurse

$$
S_{i+1}=\left(A S_{i}^{-1} A+D\right)^{-1}=D^{-1}-D^{-1} A\left(S_{i}+A D^{-1} A\right)^{-1} A D^{-1} ;
$$

the solution of this recursion is exactly the steady-state inverse covariance $C_{0}^{-1}$. The key now is that the iterates $S_{i}$ remain "almost" tree-banded if the elements of $D$ vary smoothly along the tree; i.e., we can safely set all but $O(N)$ of the elements of $S_{i}$ to zero without incurring much numerical error ${ }^{7}$. In particular, we find that the limit $S_{\infty}=C_{0}^{-1}$ remains effectively tree-banded in this case.

Now, if $S_{i}$ is tree-banded, then so is $\left(S_{i}+A D^{-1} A\right)$, as is the Cholesky decomposition $L_{i}$ defined by

$$
L_{i} L_{i}^{T}=\left(S_{i}+A D^{-1} A\right) .
$$

Similarly, since $S_{i+1}$ and $D^{-1}$ are both tree-banded, then so is $L_{i}^{-1} A D^{-1}$, and therefore we can compute this term - and from here complete the recursion for $S_{i+1}$ - in $O(N)$ time. Once the limit of this recursion (i.e., our approximation to $C_{0}^{-1}$ ) is obtained, we may apply the $O(N)$ methods described in section 2 with no further modifications. We have found that a very effective initialization for this procedure is to set

$$
S_{0}=D^{-1 / 2}\left(I-A^{2}\right) D^{-1 / 2}
$$

this may be computed in $O(N)$ time and gives the exact solution $C_{0}^{-1}$ in the case that $D$ is proportional to the identity. See Fig. 10 for an illustration.

\footnotetext{
${ }^{7}$ We do not have a good theoretical explanation for this fact; this is a purely empirical observation. Indeed, we have found that if the noise scale $D$ varies discontinuously along the tree, then $S_{i}$ tends to be much less sparse, sharply reducing the efficacy of this procedure.
} 

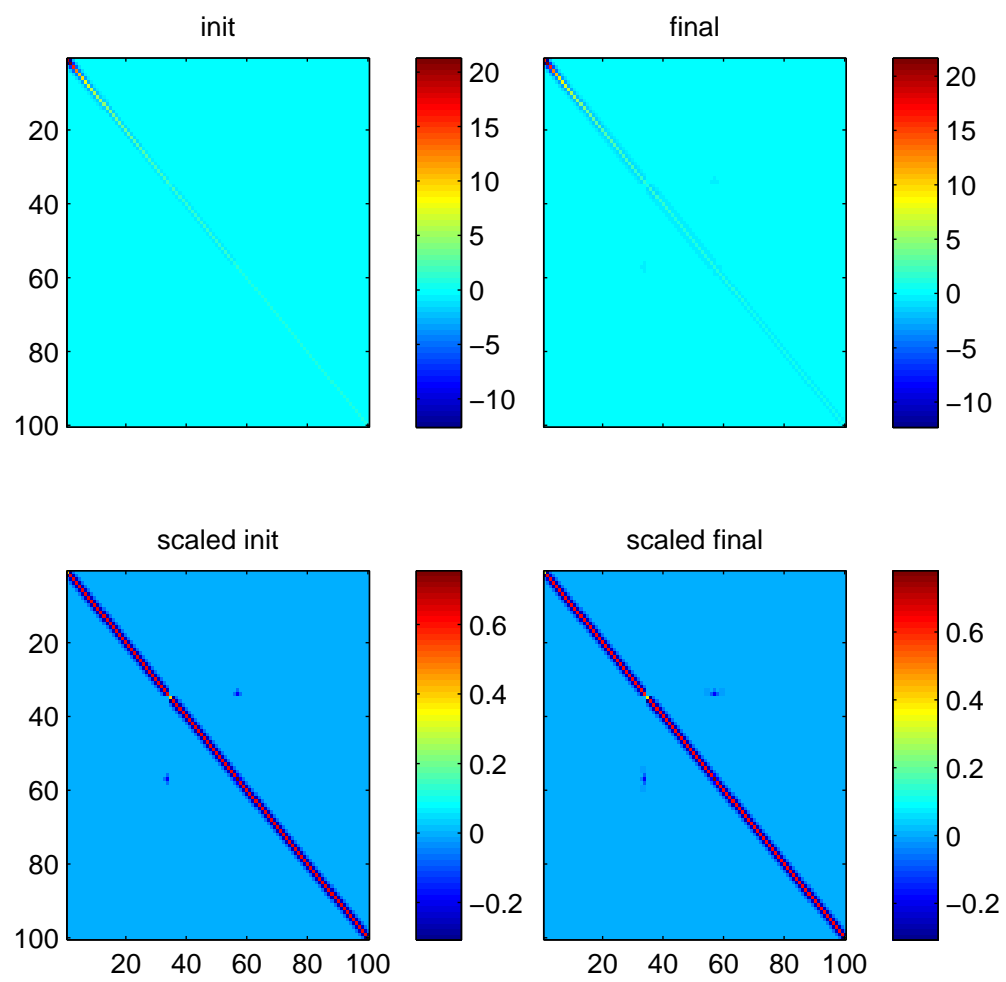

Figure 10: Computing $C_{0}^{-1}$ via the sparse recursion (9). In this case, the tree was a simple single-branched structure (with the branch at compartment 35), and the noise variance $D$ varied linearly over two orders of magnitude as a function of the compartment number. Left: initial approximate inverse covariance $S_{0}$ (top; eq. (10)) and rescaled matrix $D^{1 / 2} S_{0} D^{1 / 2}$ (bottom), included for clarity. Right: limit matrix $S_{\infty}=C_{0}^{-1}$ (top) and rescaled matrix $D^{1 / 2} S_{\infty} D^{1 / 2}$ (bottom). Note that $S_{0}$ provides a very good initialization, and that both $S_{0}$ and $C_{0}^{-1}$ are very sparse.

A very similar problem arises in the case of "quasi-active" membranes (Koch, 1984; Manwani and Koch, 1999; Coombes et al., 2007; Kellems et al., 2009), in which the dynamics of each compartment are described by a $k$-dimensional linear system (instead of the onedimensional stable dynamics for each compartment we have used in the rest of this paper); this linear system is derived from a first-order expansion about a fixed point of the original nonlinear model neuronal dynamics. In this setting the dynamics matrix $A$ may be written most conveniently as a block-tree-tridiagonal matrix, with block size equal to $k$; see, e.g., (Kellems et al., 2009) for further details. The problem is that $A$ here will be asymmetric in general, and therefore, once again, we can not solve the Lyapunov equation directly by computing the infinite matrix sum (4) explicitly. However, we have found that this quasiactive case may be handled in a very similar manner; we simply recurse (9), with $S_{0}$ set to $D^{-1 / 2}\left(I-A A^{T}\right) D^{-1 / 2}$ (equation (10) needs to be modified slightly since $A$ is asymmetric here). As in the asymmetric-noise case discussed above, we find empirically that $S_{i}$ remains tree-banded, making the recursion (9), and therefore the computation of $C_{0}^{-1}$, quite tractable. 


\subsection{Nonlinear voltage or calcium observations}

The last extension we consider involves the case of nonlinear observations $y_{t}$. For example, some voltage sensors saturate somewhat over their dynamic range, rendering the linear model $y_{t}=B_{t} V_{t}+\eta_{t}$ inadequate. It is also worth considering observations of fluorescent calcium sensors: as discussed above, voltage-sensing techniques are currently hampered by low SNR (although optimal filtering methods can at least partially alleviate this problem). On the other hand, spatiotemporal calcium-sensing fluorescence signals may be currently recorded at relatively high SNR (Gobel and Helmchen, 2007; Larkum et al., 2008), but calcium signals, by their nature, provide only highly-thresholded information about the underlying voltage, since calcium channels are inactive at hyperpolarized voltages. Can we exploit these high-SNR superthreshold calcium observations to reconstruct (at least partially) the subthreshold voltage signal? More generally, we would like to optimally combine calcium and voltage measurements, where voltage measurements may be available via imaging techniques, or whole-cell patch recordings at the soma or apical dendrite, or even through dense multielectrode recordings (Petrusca et al., 2007).

To incorporate calcium observations into our Kalman filtering framework, we must first write down a dynamical model for the evolution of the intracellular calcium concentration. For simplicity, we consider a first-order model for these calcium dynamics:

$C_{t+d t}(x)=C_{t}(x)+\left(-\frac{C_{t}(x)}{\tau_{C}}+k\left[C_{t}(x+d x)-2 C_{t}(x)+C_{t}(x-d x)\right]+f\left[V_{t}(x)\right]\right) d t+\sigma_{C} \sqrt{d t} \epsilon_{t}(x)$,

where $C_{t}(x)$ denotes the calcium concentration in compartment $x$ at time $t, k$ is a diffusion constant, $k\left[C_{t}(x+d x)-2 C_{t}(x)+C_{t}(x-d x)\right]$ represents diffusion within the dendrite (this assumes that $x$ corresponds to an interior compartment of a linear segment of the dendrite, and may be easily modified in the case of a boundary or branching compartment), and $f(V)$ is a nonlinear function corresponding to voltage-dependent calcium influx. (Note that we have approximated the calcium channel dynamics as instantaneous here, though this assumption can be relaxed.)

Now the important point is that the intracellular and transmembrane calcium diffusion terms $k$ and $1 / \tau_{C}$ are relatively small; in the subthreshold regime the calcium concentration changes much more slowly than the voltage. We can take advantage of this fact: if we sample sufficiently rapidly along the dendritic tree, then we may obtain $C_{t}(x)$ (up to some observation noise) and then numerically subtract the estimated $d C_{t}(x) / d t$ from the linear terms on the right hand side of our dynamics equation to obtain, finally, our observation $y_{t}$, which will correspond to a nonlinear measurement $f\left[V_{t}(x)\right]$ of the voltage. Of course, this will be a noisy measurement; the variance of this noise can be computed given the variance of the dynamics noise $\epsilon$ and the calcium-sensitive observation fluorescence noise. More precisely, if $y_{t}=B C_{t}+\eta_{t}$, and $k$ and $1 / \tau_{C}$ are both small, then we may approximate

$$
y_{t+d t}-y_{t} \approx B\left(f\left(V_{t}\right) d t+\sigma_{C} \sqrt{d t} \epsilon_{t}\right)+\eta_{t+d t}-\eta_{t} .
$$

If $\eta_{t}$ and $\epsilon_{t}$ are approximately Gaussian, then if we define the new observation $y_{t}^{\prime}=y_{t}-y_{t-d t}$, we can write

$$
y_{t}^{\prime} \approx h\left(V_{t}\right)+\epsilon_{t}^{\prime},
$$

for a suitable function $h($.$) and Gaussian noise \epsilon_{t}^{\prime}$ with a suitable covariance matrix. (We note in passing that a similar approach may be suitable for sodium-sensitive imaging data (Kole et al., 2008).) 
Thus, more generally, we would like to incorporate observations $y_{t}$ obeying some arbitrary conditional density $p\left(y_{t} \mid V_{t}\right)$ into our filter equations. This is of course difficult in general, since if $p\left(y_{t} \mid V_{t}\right)$ is chosen maliciously it is clear that our posterior distribution $p\left(V_{t} \mid Y_{1: t}\right)$ may be highly non-Gaussian, and our basic Kalman recursion will break down. However, if $\log p\left(y_{t} \mid V_{t}\right)$ is a smooth function of $V_{t}$, it is known that a Gaussian approximation to $p\left(V_{t} \mid Y_{1: t}\right)$ will often be fairly accurate (Fahrmeir and Tutz, 1994; Brown et al., 1998; Paninski et al., 2009), in which case our Kalman recursion may be adapted in a fairly straightforward manner.

For simplicity, we will focus on the case that the observations $y_{i t}$ are independent samples from $p\left(y_{i t} \mid B_{i} V_{t}\right)$, where $B_{i}$ denotes the $i$-th row of the observation matrix $B$. We approximate the posterior mean $\mu_{t}$ with the maximum a posteriori (MAP) estimate,

$$
\begin{aligned}
\mu_{t} & \approx \arg \max _{V_{t}}\left[\log p\left(V_{t} \mid Y_{0: t-d t}\right)+\log p\left(y_{t} \mid V_{t}\right)\right] \\
& \approx \arg \max _{V_{t}}\left[-\frac{1}{2}\left(V_{t}-A \mu_{t-d t}\right)^{T} P_{t}^{-1}\left(V_{t}-m_{t}\right)+\sum_{i} \log p\left(y_{i t} \mid B_{i} V_{t}\right)\right] .
\end{aligned}
$$

(Recall that the one-step covariance matrix $P_{t}$ and mean $m_{t}$ were defined in eqs. (7-8).) This MAP update is exact in the linear-Gaussian case (and corresponds exactly to the Kalman filter), but is an approximation more generally.

To compute this MAP estimate, we use Newton's optimization method. We need the gradient of the log-posterior with respect to $V_{t}$,

$$
\nabla=-P_{t}^{-1}\left(V_{t}-m_{t}\right)+B^{T} f_{1}\left(V_{t}\right),
$$

and the Hessian with respect to $V_{t}$,

$$
H=-P_{t}^{-1}+B^{T} \operatorname{diag}\left[f_{2}\left(V_{t}\right)\right] B .
$$

Here $f_{1}\left(V_{t}\right)$ and $f_{2}\left(V_{t}\right)$ are the vectors formed by taking the first and second derivatives, respectively, of $\log p\left(y_{i t} \mid u\right)$ at $u=B_{i} V_{t}$, with respect to $u$. Now we may form the Newton step:

$$
\begin{aligned}
V_{\text {new }} & =V_{\text {old }}-H^{-1} \nabla \\
& =V_{\text {old }}-\left(-P_{t}^{-1}+B^{T} \operatorname{diag}\left[f_{2}\left(V_{\text {old }}\right)\right] B\right)^{-1}\left(-P_{t}^{-1}\left(V_{\text {old }}-m_{t}\right)+B^{\prime} f_{1}\left(V_{\text {old }}\right)\right) \\
& =V_{\text {old }}-\left(P_{t}-P_{t} B^{T}\left(-\operatorname{diag}\left[f_{2}\left(V_{\text {old }}\right)^{-1}\right]+B P_{t} B^{T}\right)^{-1} B P_{t}\right)\left[P_{t}^{-1}\left(V_{\text {old }}-m_{t}\right)-B^{T} f_{1}\left(V_{\text {old }}\right)\right] \\
& =m_{t}+P_{t} B^{T}\left(-\operatorname{diag}\left[f_{2}\left(V_{\text {old }}\right)^{-1}\right]+B P_{t} B^{T}\right)^{-1} B\left[V_{\text {old }}-m_{t}-P_{t} B^{T} f_{1}\left(V_{\text {old }}\right)\right]+P_{t} B^{T} f_{1}\left(V_{\text {old }}\right)
\end{aligned}
$$

We iterate, using a backstepping linesearch to guarantee that the log-posterior increases on each iteration, until convergence (i.e., when $V_{\text {new }} \approx V_{\text {old }}$, set $\mu_{t}=V_{\text {old }}$ ). Then, finally, we update the covariance $C_{t}$ by replacing $W^{-1}$ with $-\operatorname{diag}\left[f_{2}\left(V_{t}\right)\right]$ in the derivation in section 2 . Since multiplication by $P_{t}$ requires just $O(N)$ time (and we need to compute $P_{t} B^{T}$ just once per timestep), all of these computations remain tractable.

Note that initialization of this iteration is important here (unlike in the standard Kalman case), since the $\log$ likelihood $\log p\left(y_{t} \mid V_{t}\right)$ is not necessarily concave in $V_{t}$. We have found that an effective strategy is to initialize $V_{\text {old }}$ by taking one Kalman step forward from the point $\mu_{t-d t}$, with $V_{t}(x)$ fixed at $E\left[V_{t}(x) \mid y_{x t}\right]$ (which may in turn be computed via a one-dimensional numerical integral if $B_{x}$ is spatially localized); this corresponds to treating $E\left[V_{t}(x) \mid y_{x t}\right]$ as the observed data, with the noise variance set to zero. From this initialization we iterate to convergence, as described above. See Fig. 11 for an illustration. 

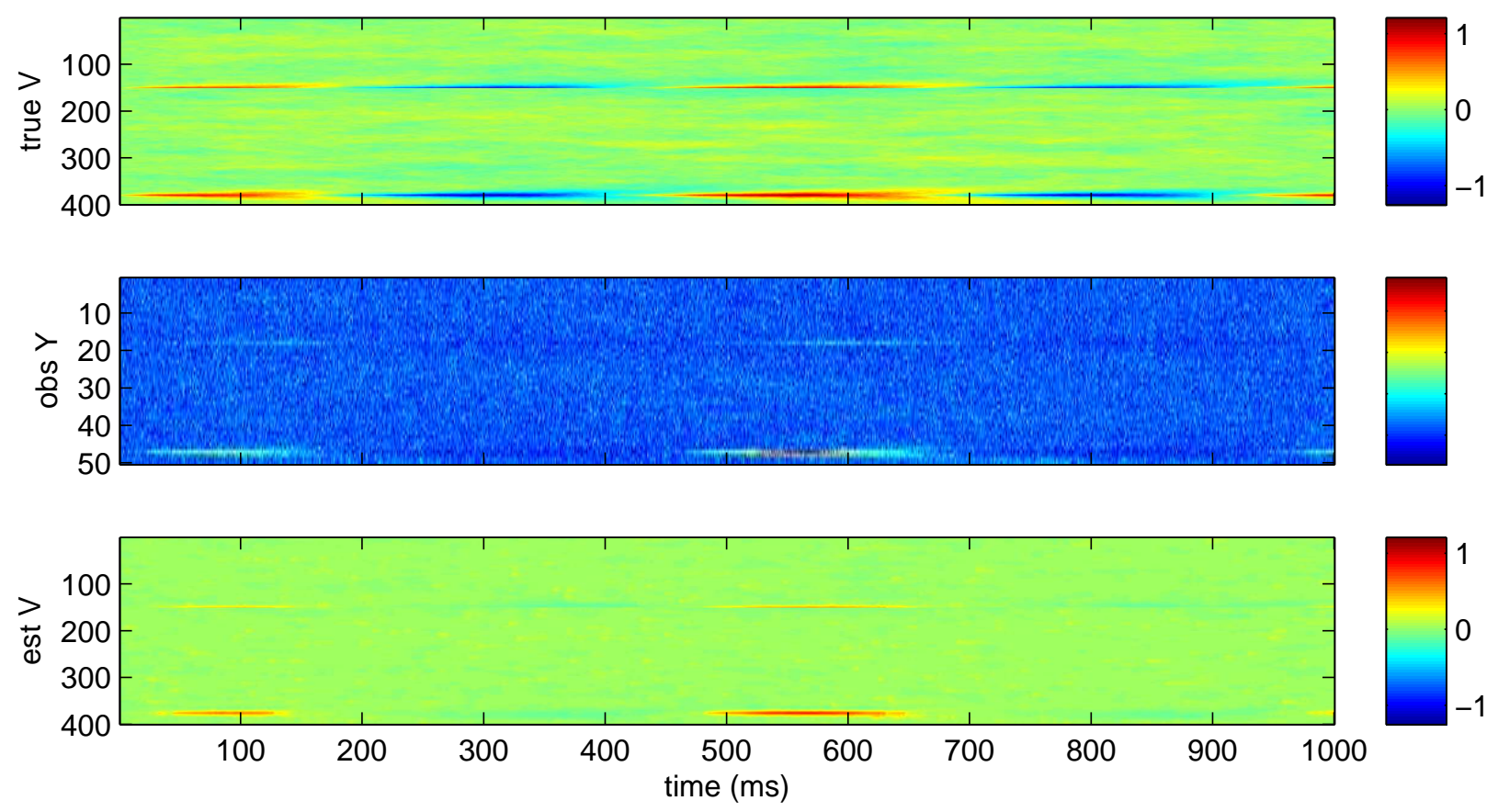

Figure 11: Example of the approximate Kalman filter applied to nonlinear observations. Top panel: true voltage $V$. Sinusoidal current $(2 \mathrm{~Hz})$ was injected into a branch point of the tree (compartment 380); the tree was a simple one-branched structure with 400 compartments in this case. Middle panel: observations $Y$ were generated by emulating the calcium-sensitive experiment described in the text. The voltage was spatially subsampled $(8 x)$, transformed by a nonlinear activation curve $f(V)$, and then corrupted with noise. Note that most details about the subthreshold voltage are obscured by this nonlinear transformation $f(V)$. Bottom panel: voltage $V$ recovered by the approximate Kalman filter. Note that superthreshold voltages are recovered fairly accurately, though subthreshold information is lost. The top and bottom panels share the same (rescaled) units.

\section{Discussion}

We have presented a collection of methods that make it possible to perform optimal quasilinear filtering of noisy, subsampled voltage observations on large realistic dendritic trees. Perhaps the major current limitation of our approach is that we are forced to assume that the dynamics on the tree are constant in time, since we make heavy use of the steady-state covariance $C_{0}$, and this quantity is well-defined only in the case of time-invariant dynamics. It will be important to generalize this approach, perhaps via extended Kalman filter or hybrid particle filter methods (Doucet et al., 2001), to the case of active dendritic membranes (Johnston et al., 1996), where the dynamics depend strongly on the recent voltage history.

Another exciting potential direction for future research involves the optimal design of sampling schemes; we would like to design our imaging experiments so that each observation reduces our posterior uncertainty about the underlying voltage $V$ (as summarized by the posterior state covariance $C_{t}$ ) as much as possible (Fedorov, 1972; Lewi et al., 2009). As one 
example, it is intuitively plausible that observations of the voltage at or near branch points may be more informative than observations at the tips of dendrites (though we have not yet checked this intuition in numerical detail). Given a limited budget of compartments that may be sampled per unit of experimental time, it might be beneficial to optimize our experimental design accordingly, by computing the expected reduction in the uncertainty $C_{t}$ for each of a number of candidate sampling schemes, using the fast methods proposed here.

Our methods are based on low-rank approximations of the time-varying system covariance $C_{t}$ (or more precisely, we represent $C_{t}$ as a low-rank perturbation of the steady-state covariance $C_{0}$ ). A very promising related approach was recently described by (Kellems et al., 2009); these authors used tools from the applied mathematical literature on low-rank approximations of high-dimensional linear systems (Antoulas, 2005) to sharply reduce the dimensionality of quasi-active models used to describe subthreshold voltage dynamics. Our method is different: we do not truncate the system dynamics directly, but instead use the full dynamics to propagate a truncated approximation to the system covariance $C_{t}$. It will be interesting in the future to combine the benefits of these two approaches.

\section{Acknowledgments}

LP is supported by a McKnight Scholar award and an NSF CAREER award. I thank Q. Huys, K. Rahnama Rad, and J. Vogelstein for many helpful conversations.

\section{References}

Antoulas, A. (2005). Approximation of large-scale dynamical systems. Cambridge University Press.

Araya, R., Eisenthal, K. B., and Yuste, R. (2006). Dendritic spines linearize the summation of excitatory potentials. PNAS, 103(49):18799-18804.

Bell, J. and Craciun, G. (2005). A distributed parameter identification problem in neuronal cable theory models. Mathematical Biosciences, 194(1):1 - 19.

Bloomfield, S. and Miller, R. (1986). A functional organization of ON and OFF pathways in the rabbit retina. J. Neurosci., 6(1):1-13.

Brette, R., Piwkowska, Z., Rudolph, M., Bal, T., and Destexhe, A. (2007). A nonparametric electrode model for intracellular recording. Neurocomputing, 70:1597-1601.

Brockwell, P. and Davis, R. (1991). Time Series: Theory and Methods. Springer.

Brown, E., Frank, L., Tang, D., Quirk, M., and Wilson, M. (1998). A statistical paradigm for neural spike train decoding applied to position prediction from ensemble firing patterns of rat hippocampal place cells. Journal of Neuroscience, 18:7411-7425.

Canepari, M., Djurisic, M., and Zecevic, D. (2007). Dendritic signals from rat hippocampal CA1 pyramidal neurons during coincident pre- and post-synaptic activity: a combined voltage- and calcium-imaging study. J Physiol, 580(2):463-484.

Chandrasekar, J., Kim, I., Bernstein, D., and Ridley, A. (2008). Cholesky-based reduced-rank square-root Kalman filtering. American Control Conference, pages 3987-3992. 
Coombes, S., Timofeeva, Y., Svensson, C. M., Lord, G. J., Josic, K., Cox, S. J., and Colbert, C. M. (2007). Branching dendrites with resonant membrane: a sum-over-trips approach. Biological Cybernetics, 97(2):137-149.

Cox, S. (2004). Estimating the location and time course of synaptic input from multi-site potential recordings. Journal of Computational Neuroscience, 17:225-243.

Cox, S. and Griffith, B. (2001). Recovering quasi-active properties of dendrites from dual potential recordings. Journal of Computational Neuroscience, 11:95-110.

Cox, S. J. and Raol, J. H. (2004). Recovering the passive properties of tapered dendrites from single and dual potential recordings. Mathematical Biosciences, 190(1):9 - 37 .

Djurisic, M., Antic, S., Chen, W. R., and Zecevic, D. (2004). Voltage imaging from dendrites of mitral cells: EPSP attenuation and spike trigger zones. J. Neurosci., 24(30):6703-6714.

Djurisic, M., Popovic, M., Carnevale, N., and Zecevic, D. (2008). Functional structure of the mitral cell dendritic tuft in the rat olfactory bulb. J. Neurosci., 28(15):4057-4068.

Doucet, A., de Freitas, N., and Gordon, N., editors (2001). Sequential Monte Carlo in Practice. Springer.

Durbin, J. and Koopman, S. (2001). Time Series Analysis by State Space Methods. Oxford University Press.

Fahrmeir, L. and Tutz, G. (1994). Multivariate Statistical Modelling Based on Generalized Linear Models. Springer.

Fedorov, V. (1972). Theory of Optimal Experiments. Academic Press, New York.

Gillijns, S., Bernstein, D., and De Moor, B. (2006). The reduced rank transform square root filter for data assimilation. Proc. of the 14th IFAC Symposium on System Identification.

Gobel, W. and Helmchen, F. (2007). New angles on neuronal dendrites in vivo. J Neurophysiol, 98(6):3770-3779.

Hines, M. (1984). Efficient computation of branched nerve equations. International Journal of Bio-Medical Computing, 15(1):69 - 76.

Holekamp, T., Turaga, D., and Holy, T. (2008). Fast three-dimensional fluorescence imaging of activity in neural populations by objective-coupled planar illumination microscopy. Neuron, 57:661-672.

Howard, A. and Jebara, T. (2005). Square root propagation. Columbia University Computer Science Technical Reports, 040-05.

Huys, Q., Ahrens, M., and Paninski, L. (2006). Efficient estimation of detailed single-neuron models. Journal of Neurophysiology, 96:872-890.

Huys, Q. and Paninski, L. (2009). Model-based smoothing of, and parameter estimation from, noisy biophysical recordings. PLOS Computational Biology, 5:e1000379. 
Johnston, D., Magee, J. C., Colbert, C. M., and Cristie, B. R. (1996). Active properties of neuronal dendrites. Ann. Rev. Neurosci., 19:165-186.

Jordan, M. I., editor (1999). Learning in graphical models. MIT Press, Cambridge, MA, USA.

Kellems, A., Roos, D., Xiao, N., and Cox, S. (2009). Low-dimensional, morphologically accurate models of subthreshold membrane potential. Journal of Computational Neuroscience.

Knopfel, T., Diez-Garcia, J., and Akemann, W. (2006). Optical probing of neuronal circuit dynamics: genetically encoded versus classical fluorescent sensors. Trends in Neurosciences, 29:160-166.

Koch, C. (1984). Cable theory in neurons with active, linearized membranes. Biological Cybernetics, 50:15-33.

Kole, M., Ischner, S., Kampa, B., Williams, S., Ruben, P., and Stuart, G. (2008). Action potential generation requires a high sodium channel density in the axon initial segment. Nature Neuroscience, 11:178-186.

Larkum, M. E., Watanabe, S., Lasser-Ross, N., Rhodes, P., and Ross, W. N. (2008). Dendritic properties of turtle pyramidal neurons. J Neurophysiol, 99(2):683-694.

Lewi, J., Butera, R., and Paninski, L. (2009). Sequential optimal design of neurophysiology experiments. Neural Computation, 21:619-687.

Manwani, A. and Koch, C. (1999). Detecting and Estimating Signals in Noisy Cable Structures, I: Neuronal Noise Sources. Neural Computation, 11(8):1797-1829.

Morse, T., Davison, A., and Hines, M. (2001). Parameter space reduction in neuron model optimization through minimization of residual voltage clamp current. Society for Neuroscience Abstracts.

Palmer, L. M. and Stuart, G. J. (2006). Site of Action Potential Initiation in Layer 5 Pyramidal Neurons. J. Neurosci., 26(6):1854-1863.

Paninski, L., Ahmadian, Y., Ferreira, D., Koyama, S., Rahnama, K., Vidne, M., Vogelstein, J., and Wu, W. (2009). A new look at state-space models for neural data. Submitted.

Paninski, L. and Ferreira, D. (2008). State-space methods for inferring synaptic inputs and weights. COSYNE.

Petrusca, D., Grivich, M. I., Sher, A., Field, G. D., Gauthier, J. L., Greschner, M., Shlens, J., Chichilnisky, E. J., and Litke, A. M. (2007). Identification and characterization of a Y-like primate retinal ganglion cell type. J. Neurosci., 27(41):11019-11027.

Press, W., Teukolsky, S., Vetterling, W., and Flannery, B. (1992). Numerical recipes in C. Cambridge University Press.

Sabino, J. (2007). Solution of large-scale Lyapunov equations via the block modified Smith method. PhD thesis, Rice University. 
Sacconi, L., Dombeck, D. A., and Webb, W. W. (2006). Overcoming photodamage in secondharmonic generation microscopy: Real-time optical recording of neuronal action potentials. Proceedings of the National Academy of Sciences, 103(9):3124-3129.

Shental, O., Bickson, D., Siegel, P. H., Wolf, J. K., and Dolev, D. (2008). Gaussian belief propagation for solving systems of linear equations: Theory and application. arXiv:0810.1119v1.

Sjostrom, P. J., Rancz, E. A., Roth, A., and Hausser, M. (2008). Dendritic Excitability and Synaptic Plasticity. Physiol. Rev., 88(2):769-840.

Spruston, N. (2008). Pyramidal neurons: dendritic structure and synaptic integration. Nature Reviews Neuroscience, 9:206-221.

Stuart, G. and Sakmann, B. (1994). Active propagation of somatic action potential into neocortical pyramidal cell dendrites. Nature, 367:69-72.

Stuart, G., Spruston, N., and Häusser, M., editors (1999). Dendrites. Oxford University Press.

Treebushny, D. and Madsen, H. (2005). On the construction of a reduced rank squareroot kalman filter for efficient uncertainty propagation. Future Gener. Comput. Syst., 21:1047-1055.

Verlaan, M. (1998). Efficient Kalman filtering algorithms for hydrodynamic models. PhD thesis, TU Delft.

Vucinic, D. and Sejnowski, T. J. (2007). A compact multiphoton 3d imaging system for recording fast neuronal activity. PLoS ONE, 2(8):e699.

Weiss, Y. and Freeman, W. T. (2001). Correctness of belief propagation in gaussian graphical models of arbitrary topology. Neural Computation, 13(10):2173-2200.

Wood, R., Gurney, K., and Wilson, C. (2004). A novel parameter optimisation technique for compartmental models applied to a model of a striatal medium spiny neuron. Neurocomputing, 58-60:1109-1116. 